(C) 2018. This manuscript version is made available under the CC-BY-NC-ND 4.0 license http://creativecommons.org/licenses/by-nc-nd/4.0/

\title{
A comprehensive model for lithium-ion batteries: from the physical principles to an electrical model
}

\author{
Alberto Berrueta, Andoni Urtasun, Alfredo Ursúa, Pablo Sanchis* \\ Institute of Smart Cities, Department of Electrical and Electronic Engineering, Public \\ University of Navarre, Campus de Arrosadía, 31006 Pamplona, Spain \\ *Corresponding author. Tel.: +34 948169613; fax: +34 948169884 \\ E-mail address: pablo.sanchis@unavarra.es
}




\begin{abstract}
The growing interest in e-mobility and the increasing installation of renewable energy-based systems are leading to rapid improvements in lithium-ion batteries. In this context, battery manufacturers and engineers require advanced models in order to study battery performance accurately. A number of Li-ion battery models are based on the representation of physical phenomena by electrochemical equations. Although providing detailed physics-based information, these models cannot take into account all the phenomena for a whole battery, given the high complexity of the equations. Other models are based on equivalent circuits and are easier to design and use. However, they fail to relate these circuit parameters to physical properties. In order to take the best of both modeling techniques, we propose an equivalent circuit model which keeps a straight correlation between its parameters and the battery electrochemical principles. Consequently, this model has the required simplicity to be used in the simulation of a whole battery, while providing the depth of detail needed to identify physical phenomena. Moreover, due to its high accuracy, it can be used in a wide range of environments, as shown in the experimental validations carried out in the final section of this paper.
\end{abstract}

Keywords: Li-ion battery; equivalent circuit model; equivalent electric circuit; storage system; electrical microgrid. 
Nomenclature

Symbol

Meaning

Unit

\begin{tabular}{|c|c|c|}
\hline$a$ & Thermodynamic activity & \\
\hline$A$ & Area & $\mathrm{m}^{2}$ \\
\hline$A_{k}$ & $\begin{array}{l}\text { Interaction parameters form Redlich-Kister } \\
\text { equation }\end{array}$ & $\mathrm{J} \cdot \mathrm{mol}^{-1}$ \\
\hline$C$ & Capacity (battery) or capacitance (capacitor) & Ah or F \\
\hline CPE & Constant phase element & $\Omega$ \\
\hline$D$ & Diffusion coefficient & $\mathrm{m}^{2} \cdot \mathrm{s}^{-1}$ \\
\hline$E$ & Energy & Wh \\
\hline$E_{A}$ & Activation energy & $\mathrm{J} \cdot \mathrm{mol}^{-1}$ \\
\hline$F$ & Faraday constant & $\mathrm{s} \cdot \mathrm{A} \cdot \mathrm{mol}^{-1}$ \\
\hline$i$ & Battery current (discharging) & A \\
\hline$j$ & Imaginary unit or flux of ions & $\mathrm{m} \cdot \mathrm{s}^{-1}$ \\
\hline$k$ & Reaction rate constant & $\mathrm{m} \cdot \mathrm{s}^{-1}$ \\
\hline$l$ & Length & $\mathrm{m}$ \\
\hline$Q$ & Charge & $\mathrm{Ah}$ \\
\hline$\vec{r}$ & Radial coordinate of electrode particle & $\mathrm{m}$ \\
\hline$R$ & Gas constant & $\mathrm{J} \cdot \mathrm{mol}^{-1} \cdot \mathrm{K}^{-1}$ \\
\hline$R$ & Resistance & $\Omega$ \\
\hline$R_{p}$ & Radius of the electrode particles & $\mathrm{m}$ \\
\hline SOC & State of charge & \\
\hline$t$ & Time & $\mathrm{S}$ \\
\hline$T$ & Battery temperature & $\mathrm{K}$ \\
\hline$v$ & Voltage & $\mathrm{V}$ \\
\hline$v_{I N T}$ & $\begin{array}{l}\text { Non-ideal interactions between charged } \\
\text { particles }\end{array}$ & $\mathrm{V}$ \\
\hline$x$ & Molar fraction & \\
\hline$Y$ & Admittance & $\mathrm{S}$ \\
\hline$z$ & Excess electric charge & \\
\hline$Z$ & Impedance & $\Omega$ \\
\hline$\alpha$ & Apparent cathodic transfer coefficient & \\
\hline$\gamma$ & Activity coefficient & \\
\hline$\varepsilon$ & Relative permittivity & \\
\hline$\varepsilon_{0}$ & Vacuum permittivity & $\mathrm{F} \cdot \mathrm{m}^{-1}$ \\
\hline$\mu$ & Chemical potential & $\mathrm{J} \cdot \mathrm{mol}^{-1}$ \\
\hline$\eta$ & Efficiency & \\
\hline$\rho$ & Density & $\mathrm{kg} \cdot \mathrm{m}^{-3}$ \\
\hline$\tau$ & Time constant & $\mathrm{s}$ \\
\hline$\theta$ & Available hole in host material & \\
\hline$\Psi$ & Exponent of CPE & \\
\hline$\omega$ & Frequency & $\mathrm{rad} \cdot \mathrm{s}^{-1}$ \\
\hline
\end{tabular}

Subscript

Meaning 
Parallel connection of $R_{c t}$ and $C P E_{d l}$

Battery

Coulombic

Charge

Charge transfer process

Diffusion process

Discharge

Double layer

Energy

Electrode

At equilibrium conditions

Faradaic process

Referred to each chemical species

Referred to anode or cathode

Lithium

Membrane

Oxidant species

Ohmic phenomena

Reducing species

Solid-electrolyte interface

Host material with intercalated lithium

Unoccupied host material

Meaning

Partial negative charge

At reference conditions 


\section{1.- Introduction}

Due to the current need to decarbonize our energy sources, there is a specific focus on renewable energy plants, decentralized electricity generation, microgrids and e-mobility [1, 2]. The energy storage system is a crucial element in these plants, making it possible to balance energy generation and consumption and thus improving the interaction with the electricity grid [3, 4]. Among the different energy storage technologies available, lithium-ion batteries are becoming more popular due to their decreasing price and functional characteristics of power and energy density and long cycle life [5-7].

In this scenario of increasing use and constant technological improvement of Li-ion batteries, a battery model is a valuable tool for many research and development activities [8]. Tasks such as battery design, energy storage system sizing, and controller and power converter design are more likely to succeed when based on a suitable battery model. Battery models can be classified into electrochemical models and equivalent-circuit models. Electrochemical models use both the mathematical expressions of physical phenomena and the material microstructure to predict battery performance [9-11]. They are generally used to study a specific battery parameter and make it possible to analyze phenomena such as the relaxation of insertion cells [12], phase change and the effect of the porous structure of electrodes [13] and the optimization of electrode porosity and thickness [14]. Although they are used to improve battery design and manufacture, complex equations need to be solved, a high computational power is required, and they fail to predict the performance of a whole battery system, given the unfeasibility of taking every battery component and process into account. Equivalent-circuit models are comprehensive models that are able to predict the overall behavior of the battery. These models are focused on estimating the state of charge (SOC) and battery impedance. The approach used to estimate the SOC depends on the model application. When a model is designed for offline simulations, the SOC is estimated by ampere-hour counting [15 - 17], which integrates the battery current over time. Online battery models can measure the actual battery current and voltage, then closed-loop algorithms such as Kalman filter, Extended Kalman filter, Hauss-Hermite quadrature filter, $\mathrm{H}_{\infty}$ filter, particle filter and least squares methods are used to avoid the integral error [18 - 21]. Each algorithm offers advantages and disadvantages regarding robustness against model and measurement inaccuracies, the credibility of results and computational requirements.

With regard to impedance estimation, there are also two main approaches. On the one hand, a machine-learning algorithm can be used to calculate the battery impedance. These 
algorithms run for a training period in which they try to minimize the error between predicted and measured battery performance. The most typical learning algorithms used in these models are based on artificial neural networks [22 - 25]. These models provide excellent results regarding accuracy, which can be maintained throughout the battery lifetime by parameter correction. However, they often fail to associate the battery behavior with the underlying physical phenomena, since they are not based on physics but on mathematical optimization. On the other hand, the battery impedance can be designed by simplifying the physical equations in order to speed up the computer simulation while keeping the accuracy as high as possible [26-29]. In this case, the impedance parameters represent the primary physical processes which determine the battery performance. There is a wide range of impedance designs based on physical battery operation. A straightforward representation of the battery impedance is a series connection of a resistance to an ideal voltage source. However, this representation is seldom used due to its poor accuracy. Instead, many authors use the Randles Model, in which a number of RC circuits are added to the series resistance in order to represent dynamic battery phenomena [30-34]. Although these models can achieve better accuracy in the prediction of high-dynamic performance, this accuracy is generally lower when the slow relaxation processes need to be taken into account.

In order to improve current models, a new equivalent-circuit model in which the parameters are firmly related to the underlying physical phenomena is proposed in this paper. The simplifications of the physical equations make it possible to integrate it in advanced electrical system models and to simulate the battery during real operation. Therefore, the model can also be used to size a battery for a particular electrical system and to improve the battery SOC control strategy. Thanks to the proposed impedance design, the accuracy of the model is guaranteed even at high-frequency operation, which allows the analysis of the interaction between the battery and the power converter connected to it. Moreover, the parameters design proposed in this model and their relationship with the physical phenomena can be used to identify the reason for particular battery shortcomings and to optimize the design and manufacturing of $\mathrm{Li}$-ion batteries. This approach is also useful for a battery user, since the model allows the intelligent use of the battery, extending its lifetime and maximizing its characteristics. A fitting procedure is also proposed to fit the model parameters to the performance of a particular battery. By way of example, this fitting procedure is applied to a commercial graphite-NMC battery, and the model is then experimentally validated for three different operating modes. These validations cover the entire battery operating range 
according to the manufacturer's specifications, that is, temperature between $0{ }^{\circ} \mathrm{C}$ and $60{ }^{\circ} \mathrm{C}$, discharge current lower than $5 \mathrm{C}$ and charge current lower than $2 \mathrm{C}$.

The aging modeling is not explicitly addressed in this paper, given that the time scale of phenomena analyzed (fractions of a second to several days) is too short for aging effects to be noticeable. Even though, batteries at different aging states can be characterized and modeled with the proposed methodology. This analysis would require the battery parameters to be fitted at each state of health of the battery.

The paper is organized as follows. A theoretical compilation of the electrochemical phenomena involved in the battery performance is presented in Section 2, explicitly covering the equilibrium potential, ohmic phenomena, double layer behavior, kinetics of the chemical reaction, ion transport and solid-electrolyte interface. Then, in order to represent these electrochemical phenomena as an equivalent-circuit model, some simplifications are proposed in Section 3 to design the battery model. The commercial battery used to illustrate the model and to validate its accuracy is described in Section 4. In Section 5, the proposed methodology to fit the model parameters, which consists in three types of experiments that can be easily performed in a typical electrical laboratory, is explained and applied to the aforementioned battery. A summary table with the value of each parameter involved in the battery model is also provided in this section. Finally, in Section 6, the performance of the model is experimentally validated for different operating temperatures, power outputs, and frequencies. The suitability of the proposed model to predict the battery performance is also experimentally tested in a real microgrid installed at the Public University of Navarre (UPNA) with satisfactory results.

\section{2.- Physico-chemical phenomena that determine the battery performance}

\subsection{Equilibrium potential of insertion electrodes}

The electrochemical reaction at the electrode-electrolyte interphase can be expressed as follows:

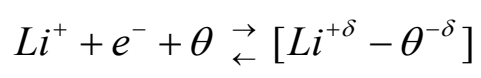

where the expression $\left[\mathrm{Li}^{+\delta}-\theta^{-\delta}\right]$ represents the intercalated species, denoted hereafter by subscript $\alpha$. 
The following relationship can be established based on thermodynamic principles:

$F \cdot U=\mu_{L i}^{0}+\mu_{\beta}-\mu_{\alpha}$

Note that the chemical potential $\mu_{i}$ ( $i=\alpha$, intercalated, or $\beta$, deintercalated species $)$ is not dependent on the composition of the host material. The chemical potential of a species $i$ in any condition can be related to the potential in other temperature and concentration conditions through activity coefficient $\left(\gamma_{i}\right)$ and the molar fraction $\left(x_{i}\right)$ [34]. In a Li-ion battery, the forces caused by the interaction between positively-charged Li-ions and negatively-charged host material cause the non-ideal behavior [35]. Therefore, activity $a_{i}$, is defined as follows, being $i$ $=\alpha$ or $\beta$ :

$a_{i}=\gamma_{i} \cdot x_{i}$

Therefore, the chemical potential can be calculated from the reference value $\left(\mu_{i}{ }^{0}\right)$, which is tabulated, through Eq. (3):

$$
\mu_{i}=\mu_{i}^{0}+R \cdot T \cdot \ln \left(a_{i}\right)
$$

Substituting Eq. (3) in (1):

$$
F \cdot U=\mu_{L i}^{0}+\mu_{\beta}^{0}-\mu_{\alpha}^{0}+R \cdot T \cdot \ln \left(\frac{x_{\beta}}{x_{\alpha}}\right)+R \cdot T \cdot \ln \left(\frac{\gamma_{\beta}}{\gamma_{\alpha}}\right)=F \cdot U^{0}+R \cdot T \cdot \ln \left(\frac{x_{\beta}}{x_{\alpha}}\right)+R \cdot T \cdot \ln \left(\frac{\gamma_{\beta}}{\gamma_{\alpha}}\right)
$$

being $U^{0}$ the equilibrium potential corresponding to the reference chemical potentials $\mu_{\alpha}{ }^{0}$ and $\mu_{\beta}{ }^{0}$. The deviation from ideal behavior, represented by the last term of Eq. (4), is due to the pseudo binary interactions between Li ions and the host matrix during intercalation and deintercalation processes. In the specific case where $\gamma_{i}=1$, Eq. (4) is called Nernst Equation.

Since $\gamma_{i}$ is related to Gibbs energy [36, 37], the Redlich-Kister equation can be used to obtain the following expression, where the summation term represents the non-ideal interactions $\left(v_{I N T}\right)$ :

$$
\begin{aligned}
& F \cdot U_{j}=F \cdot U_{j}^{0}+R \cdot T \cdot \ln \left(\frac{1-x_{\alpha, j}}{x_{\alpha, j}}\right)+\sum_{k=0}^{N} A_{k} \cdot\left[\left(2 \cdot x_{\alpha, j}-1\right)^{k+1}-\frac{2 \cdot x_{\alpha, j} \cdot k \cdot\left(1-x_{\alpha, j}\right)}{\left(2 \cdot x_{\alpha, j}-1\right)^{1-k}}\right] \\
& U=U^{0}+\frac{R \cdot T}{F} \cdot \ln \left(\frac{1-x_{\alpha, j}}{x_{\alpha, j}}\right)+v_{I N T, j}
\end{aligned}
$$

Thus, the equilibrium potential of a cell is: 
$v_{e q}=U_{c}-U_{a}$

By substituting Eq. (6) in (7) an expression of the equilibrium voltage can be obtained:

$v_{e q}=U_{c}^{0}-U_{a}^{0}+\frac{R \cdot T}{F} \cdot \ln \left(\frac{\left(1-x_{\alpha, c}\right) x_{\alpha, a}}{x_{\alpha, c} \cdot\left(1-x_{\alpha, a}\right)}\right)+v_{I N T, c}-v_{I N T, a}$

\subsection{Ohmic phenomena}

Ohmic losses represent the voltage drop due to the transfer of electrons in the electric circuit and the movement of ions through the electrolyte and membrane. These phenomena are determined, on the one hand, by the electronic conductivity of the electrodes and current collectors (usually copper and aluminum) and, on the other hand, by the ionic conductivity of the electrolyte and membrane. Since the electrolyte does not store energy, its properties are unchanged for any value of SOC. However, the variation in the electrode lithium content leads to a conductivity change, making the ohmic phenomena SOC dependent. With regard to temperature, there are opposite effects over electronic and ionic conductivity. The electronic conductivity is lower for increasing temperature, whereas the ionic conductivity increases with higher temperature. Given that the effect of ionic conductivity is more significant than that of electronic conductivity [38], ohmic losses decrease when the temperature increases.

\subsection{Polarizable electrodes}

\subsubsection{Double layer effect}

The current managed by a battery can be divided into a faradaic $\left(i_{F}\right)$ and a capacitive component, also called non-faradaic or double layer current $\left(i_{d l}\right)$ :

$i=i_{F}+i_{d l}$

In the previous equation, $i_{F}$ is the current generated by the redox reaction, while $i_{d l}$ is a consequence of differences in the Fermi levels of the electrode and electrolyte. These differences induce a slight current flow in one direction or the other, thereby creating an electric field in the area of the electrolyte closest to the electrode. Meanwhile, the excess charge in the electrode metal material is located on the contact surface. The relationship 
between charge $(z)$ and the potential gradient in the electrode $\left(\frac{d^{2} U_{j}}{d l^{2}}\right)$ is represented by Poisson Equation [39]:

$\frac{d^{2} U_{j}}{d l^{2}}=-\frac{1}{\varepsilon \cdot \varepsilon_{0}} \cdot \sum z_{i} \cdot F \cdot c_{i}^{0} \cdot \exp \left(-\frac{z_{i} \cdot F \cdot U_{j}}{R \cdot T}\right)$

where the sum refers to the local species. Eq. (10) can be solved [39], giving an expression for this distributed capacitance, also known as double layer capacitance, $C_{d l}$ :

$C_{d l}=A_{S E I} \cdot\left[\frac{2 \cdot z^{2} \cdot F^{2} \cdot \varepsilon \cdot \varepsilon_{0} \cdot c_{i}^{0}}{R \cdot T}\right]^{1 / 2} \cdot \cosh \left(\frac{z \cdot F \cdot U_{j}}{2 \cdot R \cdot T}\right)$

\subsubsection{Kinetics of chemical reaction: Butler-Volmer Equation}

The kinetics of a chemical reaction can be expressed by the well-known Butler-Volmer Equation:

$i_{F}=F \cdot A_{S E I} \cdot k^{0} \cdot\left[C_{0}(0, t) \cdot \exp \left(-\alpha \cdot f \cdot\left(v_{\text {elec }}-v_{\text {eq }}\right)\right)-C_{R}(0, t) \cdot \exp \left((1-\alpha) \cdot f \cdot\left(v_{\text {elec }}-v_{\text {eq }}\right)\right)\right]$

where $A_{S E I}$ stands for the area of the electrode-electrolyte interphase, $v_{e q}$ is the equilibrium electrode potential, $v_{\text {elec }}$ the electrode potential when subject to a current and $\alpha$ the apparent cathodic transfer coefficient.

\subsection{Ion transport}

Diffusion phenomena are described by Fick's laws of diffusion, which relate the diffusive flux to the concentration. The first law, Eq. (13), states the relationship assuming steady state; while the second law, Eq. (14), predicts how diffusion causes the concentration to change with time.

$j=-\rho \cdot D \cdot \nabla x$

$\frac{\partial x}{\partial t}=D \cdot \Delta x$

Fick's diffusion coefficient $D$ depends on the temperature, as expressed by the following equation: 
$D_{s}=D_{0} \cdot \exp \left(-\frac{E_{A}}{R \cdot\left(T-T_{0}\right)}\right)$

Diffusion phenomena have two main effects on the performance of a Li-ion battery. On the one hand, the ion diffusion through the crystal lattice in the electrodes creates a difference between the ion concentration at the electrode-electrolyte interphase, and the average electrolyte concentration, $\overline{c_{L i}}$. Given the fact that the electrochemical reaction takes place on the interphase, $v_{e q}$ and $v_{a c t}$ are defined by the concentration on this surface. This ion concentration gradient produces a concentration voltage drop during battery operation. On the other hand, ion diffusion through the membrane also has a significant impact on the battery performance, particularly during low-temperature operation [37]. This process induces a voltage drop in the battery with characteristic dynamics, which is different from the electrode diffusion and needs to be separately modeled.

\subsection{Solid-electrolyte interface}

The solid-electrolyte interface (SEI) is a passive layer created between the electrolyte and the graphite anode, whose existence is crucial for the reversibility of Li-ion batteries. Without this SEI, the electrode and electrolyte would spontaneously react, and the battery would be destroyed. Even though the SEI is built during the first battery charge and separates the anode from the electrolyte during the whole battery life, there are irreversible side reactions during the discharge process which lead to partial decompositions. During the battery charge, this SEI needs to be re-formed, consuming Lithium ions and electrons [40].

\section{Equivalent-circuit model}

The starting point of this section is the physical laws described above. Assumptions and simplifications are proposed for the representation of these laws as an equivalent-circuit model. Finally, all the parts are grouped, and the comprehensive model is built.

\subsection{Capacity, efficiency, and state of charge}

The capacity of a battery is defined as the maximum charge which can be drawn from the battery when it is fully charged $[41,42]$. Meanwhile, there are two parameters which refer to efficiency, which are energy efficiency $\left(\eta_{e}\right)$ and coulombic efficiency $\left(\eta_{c}\right)$. Both efficiencies 
$\eta_{e}$ and $\eta_{c}$ are correlated by the charging and discharging battery voltage. Due to non-ideal processes, the charging voltage is higher than the discharging voltage, therefore $\eta_{e}<\eta_{c} . \eta_{e}$ is an essential parameter for battery users since it determines the power losses and the heat flow to be dissipated. The importance of $\eta_{c}$ lies in the irreversibility of the side reactions. These reactions involve coulombic inefficiency, irreversible loss of active material and formation of passive compounds, leading to battery aging. This parameter is also important for the battery modeling, since it is part of the SOC calculation through the Coulomb-counting method, as shown in Eq. (16):

$$
S O C=S O C\left(t_{0}\right)-\int_{t_{0}}^{t} \frac{\eta_{c} \cdot i}{C} d t
$$

The most significant physical phenomena that provoke a decrease in $\eta_{c}$ is the SEI formation, which occurs during the battery charging [40]. Based on Subsection 2.5, where the SEI decomposition is explained to depend on the discharging current and temperature, the discharging process is assumed to determine the battery coulombic efficiency. $\eta_{c}$ decreases for reduced $T$, since the SEI elasticity is lower, leading to increased rupture. Even though the relationship between side reactions rate and temperature is known to follow Arrhenius law, given that the battery temperature range is limited, the relationship between $\eta_{c}$ and $T$ is modeled as a linear function, as shown in Eq. (17). With this assumption, a simplification in the model is achieved while maintaining its high accuracy. On the other hand, the relationship between $\eta_{c}$ and current has not been widely characterized and modeled in the literature. Given that $\eta_{c}$ is related to cycle aging, we take into account a result obtained in [43]. The authors of this paper conclude that lithium-ion batteries age faster for higher currents. Therefore, $\eta_{c}$ is expected to decrease for increasing current and, for the sake of simplicity, a linear trend is also assumed, as shown in Eq. (17). Finally, there are few studies about the relationship between $\eta_{c}$ and SOC. In one of the most recent and reliable papers concerning this issue [44], an experimental method is developed to obtain a measurement error in $\eta_{c}$ lower than $10 \mathrm{ppm}$. These authors are not able to measure any SOC dependency using this experimental procedure. Therefore, no dependency of $\eta_{c}$ with SOC is considered in the following equation:

$$
\eta_{c}=\eta_{c, 0}+\eta_{c, T} \cdot T+\eta_{c, i} \cdot i
$$

\subsection{Equilibrium voltage}


The equilibrium voltage $\left(v_{e q}\right)$ of a lithium-ion battery is the voltage acquired by the battery after all the dynamic processes reach the steady state. It is determined by the equilibrium voltage of each of its electrodes, which, as stated in Eq. (8), depends on the amount of intercalated lithium ions. The fraction of intercalated ions in a lithium battery with carbon anion and nickel-manganese-cobalt oxide cathode was studied in [45]. As explained in this paper, a charged battery has a $\mathrm{LiC}_{6}$ anode $\left(x_{\alpha, a}=1\right)$ and a $\mathrm{Li}_{0.3}\left(\mathrm{Ni}_{1 / 3} \mathrm{Mn}_{1 / 3} \mathrm{Co}_{1 / 3}\right) \mathrm{O}_{2}$ cathode $\left(x_{a, c}=0.3\right)$. Meanwhile, a discharged battery has a $\operatorname{LiC}_{72}$ anode $\left(x_{a, a}=0.083\right)$ and a $\mathrm{Li}\left(\mathrm{Ni}_{1 / 3} \mathrm{Mn}_{1 / 3} \mathrm{Co}_{1 / 3}\right) \mathrm{O}_{2}$ cathode $\left(x_{\alpha, c}=1\right)$. Taking into account that the $\mathrm{SOC}$ is the relationship between the charge stored in the battery and the maximum storable charge, a linear relationship between molar fraction and SOC can be inferred. The above-mentioned limits correspond to $S O C=1$ and $S O C=0$ respectively. Thus, these relationships can be written as:

$x_{\alpha, a}=0.083+0.917 \cdot S O C$

$x_{\alpha, c}=1-0.7 \cdot S O C$

Since the measurement of anode and cathode voltage in a full operating cell cannot be achieved, some simplifications of Eq. (8) are needed in order to calculate $v_{e q}$. The equivalent terms of this equation are grouped, making it possible to fit the parameters of the expression from experimental data. For this purpose, the reference potential is defined as $U_{b a t}^{0}=U^{0}{ }_{c}-U^{0}{ }_{a}$. Besides, non-ideal interactions $v_{I N T, c}$ and $v_{I N T, a}$ are assumed to be similar and grouped in a single term, and interactions with an order higher than seven are disregarded. Therefore, the following equation is obtained, where $\mathrm{v}_{I N T}$ is a seven-order polynomial function:

$v_{e q}=U_{b a t}^{0}+\frac{R \cdot T}{F} \cdot \ln \left(\frac{\left(1-x_{\alpha, c}\right) x_{\alpha, a}}{x_{\alpha, c} \cdot\left(1-x_{\alpha, a}\right)}\right)+v_{I N T}$

\subsection{Fast-dynamic processes}

\subsubsection{Ohmic phenomena}

The ohmic phenomena detailed in Subsection 2.2 are typically represented by an SOC- and temperature-dependent series resistance $\left(R_{\text {ohm }}\right)$ because of their proportionality to the square of the battery current:

$R_{\text {ohm }}=R_{\text {ohm }, 0}+R_{\text {ohm }, T} \cdot T+R_{o h m, S O C}: S O C$ 
Since the Lithium-ion concentration in the electrodes is linearly related to SOC $($ Eq. $(18,19))$, the relationship between $R_{\text {ohm }}$ and SOC is also assumed to be linear, as shown in Eq. (21). Even though $R_{o h m}$ is known to follow Arrhenius law, a linear relationship is assumed to model this effect, which leads to a reasonably small deviation for the operating temperature range $\left(0^{\circ} \mathrm{C}-60^{\circ} \mathrm{C}\right)$. This simplification has two main advantages for the battery modeling. On the one hand, a faster computation is achieved and, on the other hand, the thermal coefficient $R_{o h m, T}$ can be calculated with a smaller test map.

\subsubsection{Charge transfer through the SEI}

The current division into its faradaic and capacitive components shown in Eq. (9) is modeled here. For the capacitive current, the double-layer capacitor expressed in Eq. (11) could be used. However, this ideal element is not able to accurately represent real systems distributed over a finite region. Instead, a constant phase element $\left(C P E_{d l}\right)$ has been herein chosen, as shown in Fig. 1. The admittance of a constant-phase element can be expressed by two frequency-independent coefficients $A_{0}$ and $\Psi$ as shown in Eq. (22) [39]:

$$
Y_{C P E}=A_{0}(j \cdot \omega)^{\Psi}
$$

This element behaves as an ideal capacitor when $\Psi=1$ and as an ideal resistor when $\Psi=0$. It is thought to arise, when $\Psi \neq 0$ or 1 , from the presence of inhomogeneity in the electrode-material system [47].

The faradaic current $i_{F}$ is determined by the reaction kinetics at the electrodes and represented in the model by a current source, as shown in Fig. 1. The Butler-Volmer equation, Eq. (12), is valid to calculate faradaic current produced by insertion reactions [48, 49]. Some minor modifications are required to use the molar fraction $x_{\alpha}$ instead of concentrations $C_{i}$ as the independent variable. Two distinct scenarios for $i_{F}$ are analysed in

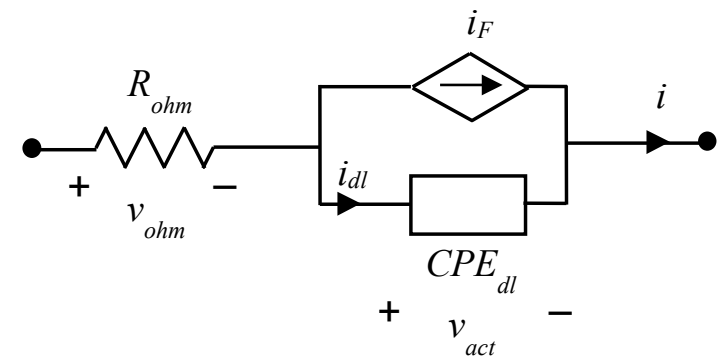

Fig. 1: Equivalent-circuit representation for the fast-dynamic processes of a Li-ion battery. For small-signal model, the current source $i_{F}$ is substituted by a resistance $R_{c t}$ as shown in Eq. (28). 
order to get simplifications of the Butler-Volmer equation. On the one hand, the widely-used simplification for large current shown in Eq. (23) is the expression implemented in the final model. The activation overpotential is defined as $v_{a c t}=v_{\text {elec }}-v_{\text {eq }}$ :

$\left.i_{F}=F \cdot A \cdot k_{0}^{0} \cdot e^{-\frac{E_{A}}{R \cdot T}} \cdot e^{\left(\frac{n \cdot F}{2 \cdot R \cdot T} \cdot v_{a c t}\right.}\right) \cdot\left(x_{\alpha, a} \cdot x_{\alpha, c}\right)^{0.5}$

On the other hand, Eq. (12) is linearized to get the small-signal model which will be useful for the parameter fitting process explained in Section 5. For small vact the Butler-Volmer Equation can be approximated as [50]:

$i_{F}=i_{0} \cdot\left[1-\alpha \cdot f \cdot v_{a c t}-1-(1-\alpha) \cdot f \cdot v_{a c t}\right]$

$i_{F}=i_{0} \cdot f \cdot v_{a c t}$

being

$i_{0}=A \cdot F \cdot k^{0} \cdot C_{O}^{(1-\alpha)} \cdot C_{R}{ }^{\alpha}$

Thus, the relationship between $i_{F}$ and $v_{a c t}$ can be considered linear if the current is not too high. The linearization is valid if $n \cdot F \cdot\left|v_{a c t}\right|<<R \cdot T$, which some authors take as $\left|v_{a c t}\right|<5 \mathrm{mV}$ [51]. This relationship is expressed by the so-called charge-transfer resistance $R_{c t}$ :

$R_{c t}=\frac{v_{a c t}}{i_{F}}=\frac{R \cdot T}{n \cdot F \cdot i_{0}}$

Assuming $\alpha=0.5$ and an Arrhenius trend in $k^{0}$, the following expression for $R_{c t}$ is obtained:

$R_{c t}=\frac{1}{\left(x_{\alpha, a} \cdot x_{\alpha, c}\right)^{0.5}} \cdot\left[\frac{R}{n \cdot F^{2} \cdot A_{S E I} \cdot k_{0}^{0}} \cdot T \cdot e^{E_{A} / R \cdot T}\right]$

In this small-signal scenario, the charge transfer process through the SEI is characterized by parameters $C P E_{d l}$ and $R_{c t} . Z_{a r c}$ is the impedance of the parallel connection of these two parameters. If $\tau=\left(A_{0} \cdot R_{c t}\right)^{1 / \Psi}, Z_{\text {arc }}$ can be expressed as:

$Z_{\text {arc }}=\frac{R_{c t}}{1+(j \cdot \omega \cdot \tau)^{\Psi}}$

Given that $R_{c t}$ depends on SOC and $T, \tau$ also has a dependency on these two variables. An exponential relationship between $\tau$ and SOC and a quadratic expression to relate $\tau$ and $T$ are empirically proposed for the temperature range analyzed in this model. Therefore, $\tau$ is defined as: 
$\tau=a+b \cdot e^{c \cdot S O C}$

being $c$ a constant parameter and $a$ a temperature-dependent coefficient as shown in Eq. (31):

$a=a_{0}+a_{1} \cdot T+a_{2} \cdot T^{2}$

\subsection{Diffusion processes}

\subsubsection{Membrane diffusion}

The diffusion of lithium ions through the membrane, described by Fick's laws (Eq. (13) and (14)), is a distributed process. Several simplifications have been proposed in the literature for this process to be included in a computational model, most of which consist of a distributed $\mathrm{RC}$ network [52]. With the aim of enhancing the functionality of the model, we use a single $\mathrm{RC}$ branch to model the membrane diffusion process, as represented in Fig. 2 (a). While $C_{d i f, \text { mem }}$ can be assumed to have a constant value, Fick's laws state an Arrhenius-type behavior for $R_{d i f, m e m}$, as shown in Eq. (32):

$$
R_{d i f, \mathrm{mem}}=K_{d i f, \mathrm{mem}} \cdot \exp \left(\frac{b_{d i f, \mathrm{mem}}}{T-T_{0, d i f, \mathrm{mem}}}\right)
$$

This diffusion process is SOC independent since the properties of the electrolyte and membrane are unchanged with variable SOC.

\subsubsection{Electrode diffusion}

Similarly, electrode diffusion is modeled by an RC branch with parameters $R_{\text {dif, elec }}$ and

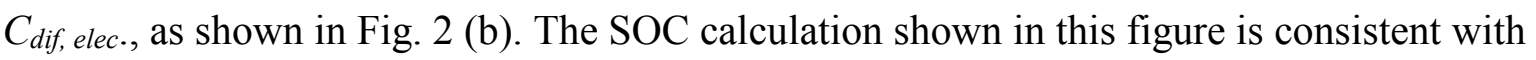
Eq. (16). The variable $S O C_{\text {sur }}$ is the state of charge corresponding to the lithium molar fraction at the electrode surface. This difference between $S O C$ and $S O C_{\text {sur }}$ results in a battery voltage drop. In order to propose an expression for $R_{d i f, \text { elec }}$ and $C_{d i f, \text { elec }}$, spherical electrode particles with radius $R_{p}$ and radial diffusion, as shown in Fig. 3, are assumptions that allow an important reduction of model complexity while keeping a high level of accuracy. This leads to the so-called single particle model, which is widely used in the literature with different modifications [53-55]. Let $D$ be the diffusion coefficient and $r$ the radial coordinate of electrode particles. Eq. (14) can be reduced to one dimension: 


$$
\frac{\partial x_{L i}}{\partial t}=\frac{D}{r^{2}} \cdot \frac{\partial}{\partial r}\left(r^{2} \cdot \frac{\partial x_{L i}}{\partial r}\right)
$$

The average volumetric Lithium concentration, which is determined by SOC, can be obtained from:

$$
\overline{x_{L i}}=\frac{\int_{0}^{R a} 4 \cdot \pi \cdot r^{2} \cdot x_{L i}(r) d r}{\frac{4}{3} \cdot \pi \cdot R_{p}{ }^{3}}
$$

The current density over the particle surface can be assumed to be uniform [53]. Besides, $x_{L i}$ during a constant-current discharge has a parabolic profile for the radial coordinate as shown in Fig. 3 [56], and can be parametrized as follows:

$$
x_{L i}(r)=a_{0}+a_{1} \cdot r+a_{2} \cdot r^{2}
$$

The boundary conditions are:

$$
\begin{aligned}
& \left.\frac{\partial x_{L i}}{\partial r}\right|_{r=0}=0 \Rightarrow a_{1}=0 \\
& \left.D \cdot \frac{\partial x_{L i}}{\partial r}\right|_{r=R_{p}}=-\bar{j}_{L i} \Rightarrow a_{2}=-\frac{\bar{j}_{L i}}{2 \cdot D \cdot R_{p}}
\end{aligned}
$$

In this context, when the steady state has been reached, Eq. (34) and (35) can be combined to provide the following expression:

$$
\begin{aligned}
& \overline{x_{L i}} \cdot \frac{4}{3} \cdot \pi \cdot R_{p}^{3}=4 \cdot \pi \cdot\left(\frac{a_{0}}{3} \cdot R_{p}^{3}+\frac{a_{2}}{5} \cdot R_{p}{ }^{5}\right) \\
& x_{L i}\left(R_{p}\right)=a_{0}+a_{2} \cdot R_{p}{ }^{2}
\end{aligned}
$$

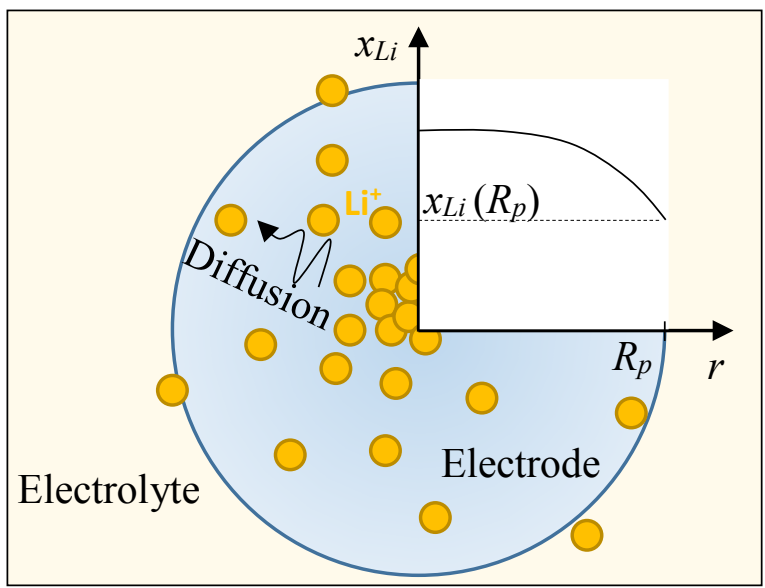

Fig. 3: Single-particle model used for the electrode diffusion. 
From the combination of Eq. (37), (38) and (39), the value of surface Li-ion concentration for a constant current and temperature discharge is inferred:

$x_{L i}\left(R_{p}\right)=\overline{x_{L i}}+\frac{R_{p}}{5 \cdot F \cdot A_{S E I}} \cdot \frac{1}{D} \cdot i \cdot \eta_{c}$

$x_{L i}\left(R_{p}\right)$ is a vital model parameter, given that it determines the battery equilibrium voltage and faradaic current. In the same way as $\overline{x_{L i}}$ is related to SOC through Eq. (18) and (19), $x_{L i}\left(R_{p}\right)$ determines the value of $S O C_{\text {sur }}$.

In order to express $R_{d i f, \text { elec }}$ as a function of $T$, the Arrhenius behavior of $D$ is included and Eq. (40) can be rewritten as:

$$
\frac{\bar{x}_{L i}-x_{L i}\left(R_{p}\right)}{i \cdot \eta_{c}}=-\frac{R}{5 \cdot F \cdot A \cdot D_{0}} \cdot e^{\frac{E_{A} / \kappa}{T-T_{0}}}
$$

Considering that $\bar{x}_{L i}$ and $x_{L i}\left(R_{p}\right)$ are related to $S O C$ and $S O C_{s u r}$ respectively, Eq. (42) can be fitted to $R_{d i f, \text { elec }}$ :

$$
\frac{S O C-S O C_{\text {sur }}}{i \cdot \eta_{c}}=R_{d i f, \text { elec }}=K_{d i f, \text { elec }} \cdot \exp \left(\frac{b_{d i f, \text { elec }}}{T-T_{0, \text { dif }, \text { elec }}}\right)
$$

Finally, an empirical expression is proposed for the temperature dependency of $C_{\text {difelec }}$. Given that polynomial expressions are easy to handle in a computer simulation, a second order polynomial is chosen, as shown in Eq. (43):

$C_{\text {dif }, \text { elec }}=a_{\text {Cdifelec }} \cdot T^{2}+b_{\text {Cdifelec }} \cdot T+c_{\text {Cdifelec }}$

\subsection{Final configuration of the model}

The above-modeled phenomena are grouped, and the complete electric model of the Li-ion battery is configured as shown in Fig. 4. This equivalent-circuit model has three main components: a voltage source for the equilibrium voltage $v_{e q}$ as described in Eq. (8), the representation of the fast-dynamic processes shown in Fig. 1 and the representation of membrane diffusion process shown in Fig. 2 (a). Besides, the circuit presented in Fig. 2 (b) is used for the calculation of the parameter $S O C_{\text {sur }}$. 
In this model, $\eta_{c}$ is calculated through Eq. (17) and used to figure out the value of SOC as stated in Eq. (16). This data is included in the electrical circuit shown in Fig. 2 (b), thereby obtaining the value of $S O C_{\text {sur }}$ by means of circuit simulation. Besides, the value of $x_{\alpha}$ on both electrode surfaces is calculated trough Eq. (18) and (19) using $S O C_{\text {sur }}$ as the state of charge. Finally, Eq. (20) is used to figure out the value of $v_{e q}$.

The dynamic phenomena included in the model are ohmic and charge-transfer behavior, as detailed in Subsection 3.3. The ohmic phenomena, represented by $R_{o h m}$, depend on $S O C$ and $T$, as stated in Eq. (21). The faradaic current is calculated as a function of $x_{L i}\left(R_{p}\right)$ and $T$, as described by the Butler-Volmer equation, particularized by Eq. (23). Moreover, $C P E_{d l}$ is, as shown in Eq. (22) and (30), a function of $T$ and SOC. Concerning membrane diffusion, represented in Fig. 4 by $R_{d i f, m e m}$ and $C_{d i f, m e m}$, the temperature dependency is confined to the parameter $R_{d i f, m e m}$, as explained in Section 3.4.1, see Eq. (32).

\section{4.- Battery description and experimental setup}

The experimental work presented hereinafter has been conducted at the UPNA Renewable Energies Laboratory with the Li-ion battery shown in Fig. 5 (a). The rated voltage of this battery pack is $133 \mathrm{~V}$ and its capacity $40 \mathrm{Ah}$. It is built by the series connection of 36 pouchtype cells, with graphite anodes and NMC cathodes, with a maximum current of 200 A during discharging and 80 A during charging. The temperature which guarantees the safe operation of the battery ranges from $0{ }^{\circ} \mathrm{C}$ to $60^{\circ} \mathrm{C}$. With the study of such a battery pack, all the components of the battery (connections, safety elements, and so forth.) are included, which increases the pertinence of the model to predict the performance of the entire battery system.

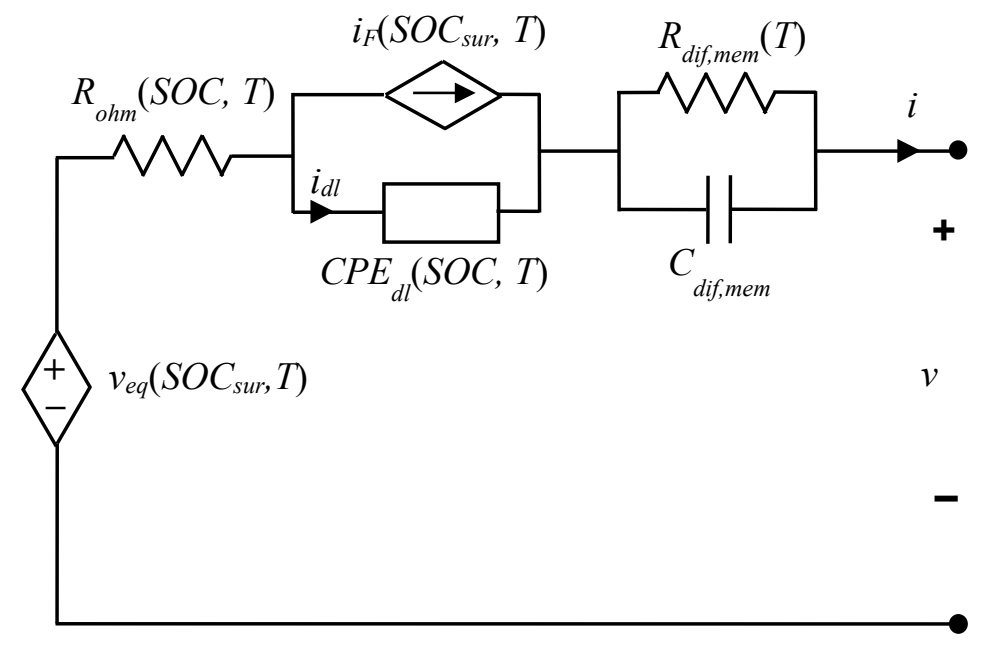

Fig. 4: Battery equivalent-circuit model. 
The cells of this battery are grouped into six modules, each comprising six cells, as can be seen in Fig. 5 (a). Moreover, the battery has measurement and communication circuits to be controlled by a BMS, even though a BMS has not been used for the experiments presented herein. We were, therefore, able to do experiments under extreme operating conditions and to equalize the cells whenever needed. The studied battery is housed in a climate chamber, as shown in Fig. 5 (b). Outside the climate chamber, there are a DC power supply (SPS400 x 75k12D, Amrel), two electronic loads (PLA4K-400-360-I and PLA7.5K-600-400), a data logger (WT1800, Yokogawa). These elements are used to measure current, voltage and ambient temperature. A digital oscilloscope (TDS 5034, Tektronix), and the battery communication software are also available to measure the voltage and temperature of each cell. The current profile supplied or drawn from the battery is externally programmed from a PC through a digital signal processor (DSP, DS 1104, dSpace). A frequency response analyzer (FRA, Amrel) is also used in combination with the electronic load in order to perform electrochemical impedance spectroscopy (EIS) tests.

\section{5.- Experimental design to obtain the model parameters}

\subsection{Test procedure}

The process to obtain the parameters for the battery model shown in Fig. 4 is detailed in this section. With the aim of ensuring test uniformity of the experiments, the following initialization protocol is followed. Prior to each experiment, the battery is kept in idle state for 12 hours with a SOC of around 0.8 . The cells are subsequently equalized to obtain a voltage

(a)

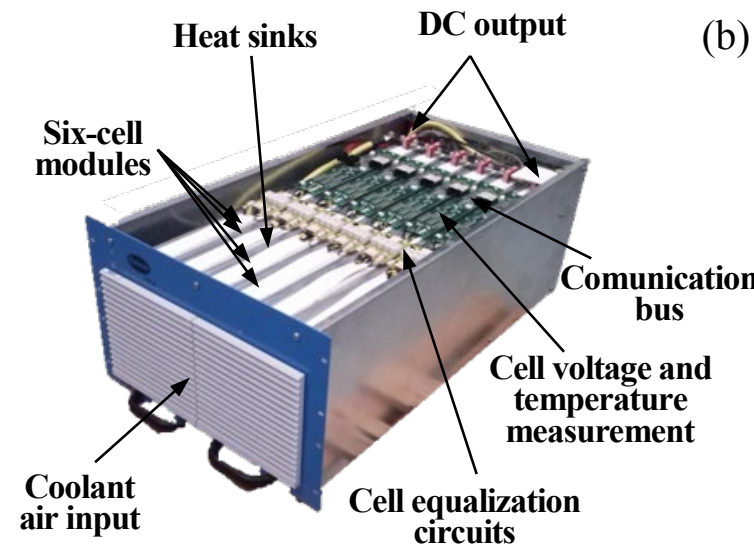

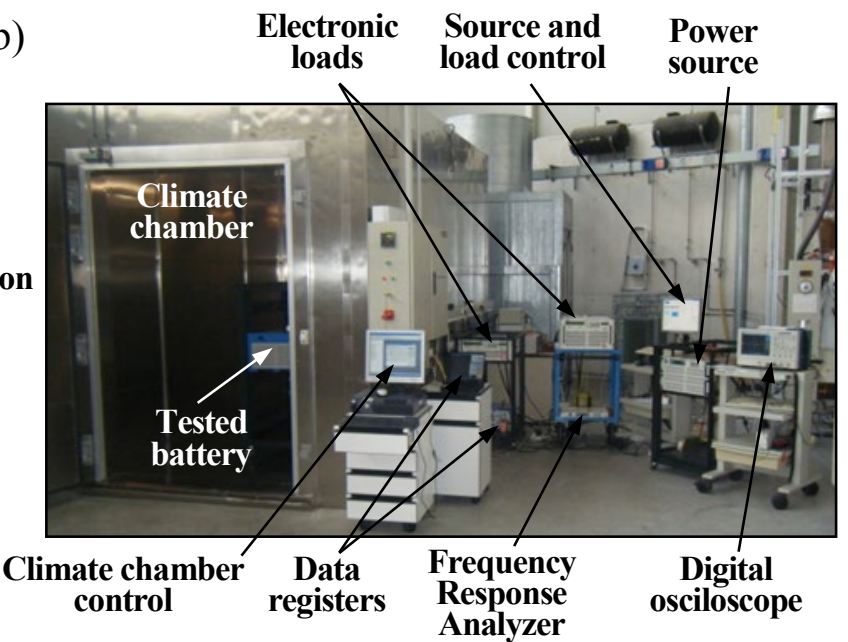

Fig. 5: Laboratory equipment: the studied battery, with the top cover open (a) and battery test bench with the battery inside the climate chamber (b). 
dispersion of less than $20 \mathrm{mV}$. Then, the battery is charged following a CC-CV protocol with $i=1 \mathrm{C}$ until the current decreases in the $\mathrm{CV}$ zone to $i=0.05 \mathrm{C}$. This point is considered as the beginning of the test. For the sake of comparability with other studies, C-rate is used as battery current unit through the whole paper. It is defined as the current that, continuously extracted from a fully charged battery, provokes the complete battery discharge. Given that the battery tested in this paper has a capacity of $40 \mathrm{Ah}, i=1 \mathrm{C}$ means $i=40 \mathrm{~A}$.

Three types of experiments are conducted in this paper: type $a, b$ and $c$. Type $a$ experiments consist of a full constant-current discharge followed by a $\mathrm{CC}-\mathrm{CV}$ standard charge in which $i=1 \mathrm{C}$. Type $b$ experiments consist of a stepped charge and discharge, with current steps of a few minutes and $i=1 \mathrm{C}$. Between the current steps, there are resting periods with a duration of 60 minutes. Finally, type $c$ experiments comprise electrochemical impedance spectroscopy (EIS). The tests mentioned above are conducted with different ambient temperatures covering the whole range of the battery operating temperatures $\left(0{ }^{\circ} \mathrm{C}-\right.$ $\left.60^{\circ} \mathrm{C}\right)$.

Based on these experiments, a five-stage fitting method is developed to calculate the parameters of the equivalent circuit. The first stage concerns the calculation of the battery capacity (Subsection 5.2). Secondly, $v_{e q}(S O C)$ is fit in Subsection 5.2. The parameters characterizing the battery impedance are then calculated in Subsection 5.3. In Subsection 5.4, the diffusion parameters of the membrane are fit to experimental data and, finally, the diffusion process in the electrodes is studied in Subsection 5.4.

\subsection{Capacity, efficiency and equilibrium voltage}

The first variable to be measured was battery capacity $C$. For this purpose, a type $a$ experiment with negligible influence of the battery impedance is used. The influence of the impedance is minimized for low currents and high temperatures. Therefore, for the test used for capacity measurement, $i=0.5 \mathrm{C}$, and $T=47.5 \pm 0.5^{\circ} \mathrm{C}$. A value of $C=41 \mathrm{Ah}$ is obtained. $\eta_{c}$ is the second parameter to be fitted. To do so, all the tests of type $a$ are used, with different values of $i$ and $T$. The result is shown in Fig. 6 (a). Note that $T$ is the battery operating temperature, which is usually higher than ambient temperature due to the heat generation associated with battery operation and the heat dissipation processes. Due to this issue, there is a point in Fig. 6 (b) whose temperature exceeds $60^{\circ} \mathrm{C}$, the maximum ambient temperature. The values for the parameters of Eq. (17) are summarized in Table 1. 
Type $b$ experiments were then used to obtain the coefficients of Eq. (20), in which $v_{e q}$ is defined. The slow diffusion phenomena are stabilized during the resting periods, allowing the accurate measurement of $v_{e q}$, as shown in Fig. 6 (b). The charging and discharging measurements are almost superimposed, proving that the $v_{e q}$ curve has no hysteresis in NMC batteries. The fitted parameters are shown in Table 1.

\subsection{Battery impedance}

The impedance of a battery is characterized by $R_{o h m}$ and $Z_{\text {arc }}$, defined in Eq. (29). In order to determine its value, type $c$ experiments (EIS) were carried out on a number of values of SOC and $T$. The frequency analyzed in the EIS experiments ranges from $100 \mathrm{mHz}$ to $10 \mathrm{kHz}$. Since ohmic and charge transfer phenomena are measurable in this frequency range, the electric circuit shown in Fig. 7 (a) can be fitted to the measured data.

Fig. 7 (b) shows the Nyquist diagram of the battery at $24.1{ }^{\circ} \mathrm{C}$ and $S O C=0.6$. For the parameter fitting, $R_{o h m}$ is firstly calculated, which is the impedance of the battery at high frequency (around $600 \mathrm{~Hz}$ ) and corresponds to the x-intercept of its impedance arc. In the example shown in Fig. 7 (b) $R_{o h m}=33.13 \mathrm{~m} \Omega$. The relationship between the real and imaginary part of the admittance in a depressed $\operatorname{arc}\left(Y_{\text {arc }}\right)$ is linear, as presented by Barsoukov and Macdonald [57]. A value for $\Psi$, Eq. (29), can be obtained from the linear fit shown in Fig. 7 (c). Moreover, $R_{c t}$ can be obtained from the $\mathrm{x}$-intercept of this fitted line, since $\operatorname{Im}\left(Y_{\text {arc }}\right)=0$ and $\operatorname{Re}\left(Y_{\text {arc }}\right)=\frac{1}{R_{c t}}$ when $\omega \rightarrow 0(s \rightarrow 0) . \tau$ is the last parameter to be calculated. Its value can be obtained from the relationship proposed in [57] between vectors $u=\overrightarrow{B P}$ and
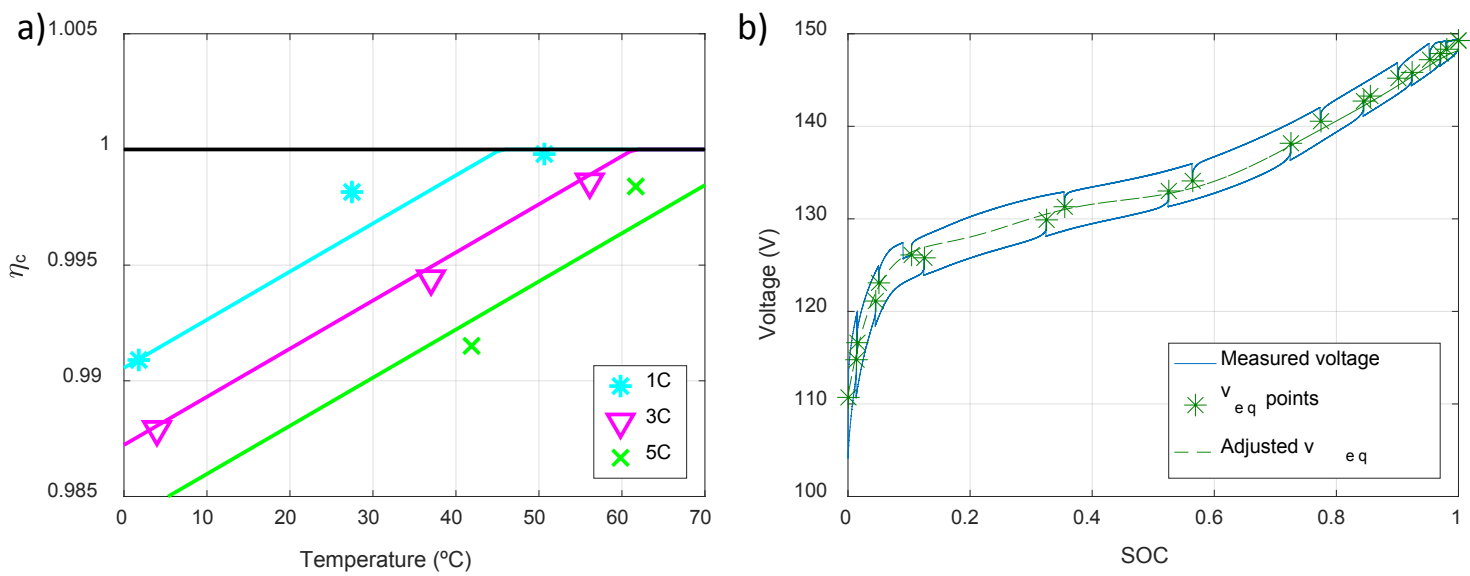

Fig. 6: Experimental data and curve fitting. $\eta_{c}$ obtained from experiments of type $a$ (a) and voltage during a type $b$ experiment (b). 
$v=\overrightarrow{P Q}$, where point $B=\left(R_{o h m}, 0\right), Q=\left(R_{o h m}+R_{c t}, 0\right)$ and $\mathrm{P}$ is a generic point in the Nyquist diagram:

$\ln \left|\frac{v}{u}\right|=\Psi \cdot[\ln (\omega)+\ln (\tau)]$

Therefore, if a linear polynomial is fitted to the relationship between $\ln |v / u|$ and $\ln (\omega)$, as shown in Fig. 7 (d), the y-intercept is an estimator of $\tau$.

This fitting process is repeated for all the type $c$ experiments. $R_{o h m}$ values obtained from the individual EIS experiments and a fitting of Eq. (21) are shown in Fig. 8 (a). The parameter values, summarized in Table 1, are the result of this fitting. On the other hand, the parameters from Eq. (28) are fitted to the data shown in Fig. 8 (b). Thereby, the value of $E_{A}=77 \mathrm{~kJ} \mathrm{~mol}^{-1}$ is obtained, consistent with previous studies which propose $E_{A}=41 \mathrm{~kJ} \mathrm{~mol}^{-1}$ for a graphite electrode [58] or $E_{A} \approx 40 \mathrm{~kJ} \mathrm{~mol}^{-1}$ for $18650 \mathrm{Li}$-ion batteries [59]. These parameters are represented in the model by $i_{F}$, defined in Eq. (23).

a)

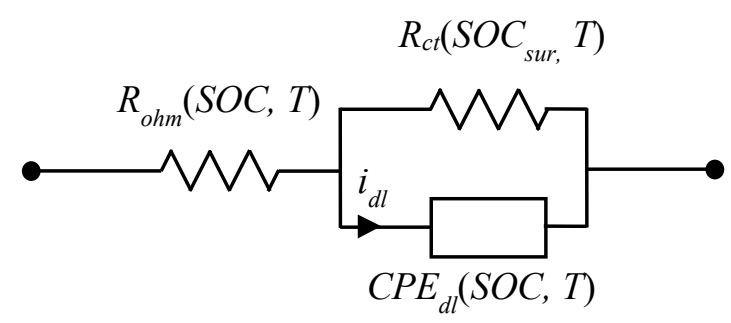

c)

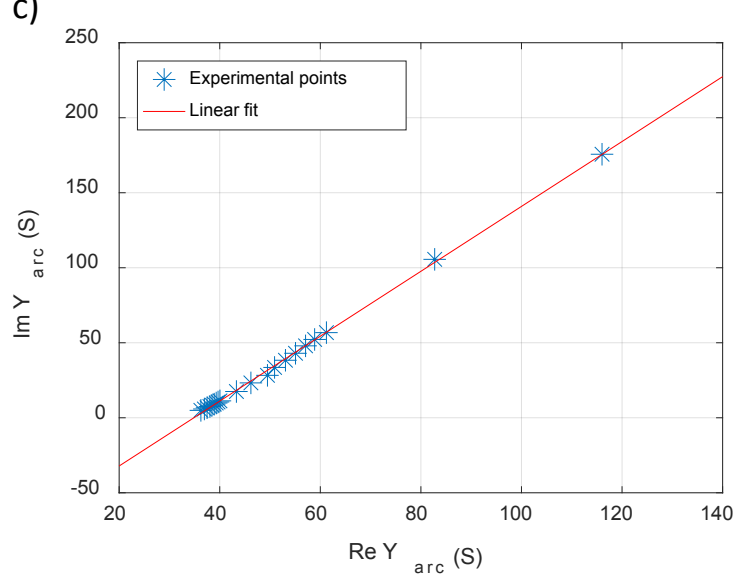

b) 20

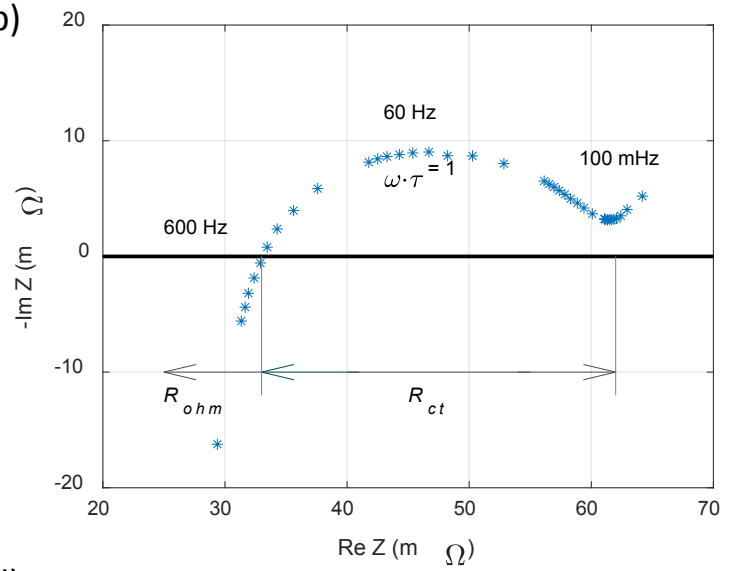

d)

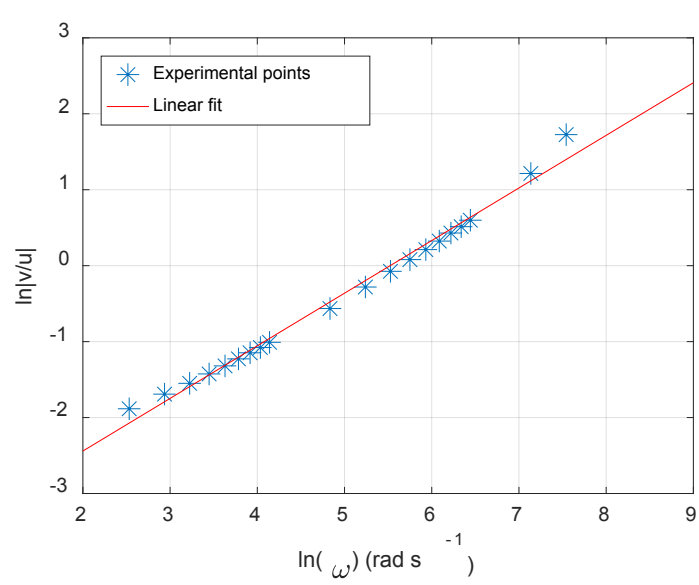

Fig 7: Fitting of EIS experiments: Equivalent circuit (a), Nyquist diagram with $T=24.1{ }^{\circ} \mathrm{C}$ and $S O C=0.6$ (b), linear fit to $Y_{\text {arc }}$ (c) and linear fit of $\ln |v / u|$ vs. $\ln (\omega)$ (d). 
The element $C P E_{d l}$ is defined by parameters $\tau$ and $\Psi$, as detailed in Eq. (29) and (30). Its measured values and the fitting of Eq. (30) are shown in Fig. 8 (c). Likewise, calculated $\Psi$ and average value are represented in Fig. 8 (d). The values of the parameters are summarized in Table 1.

\subsection{Diffusion phenomena}

The two battery components with greatest influence in the diffusion process are the membrane and the electrodes, as explained in Subsection 3.4. The vertical voltage shift arising from diffusion phenomena (see Fig. 9) is proposed to be attributed to diffusion in the membrane, while the horizontal shift is attributed to diffusion in electrodes. This differentiation is because electrode diffusion provokes a concentration gradient in electrodes and therefore a difference between the actual battery SOC and the variable $S O C_{\text {sur }}$.

The diffusion resistances $\left(R_{\text {dif, mem }}\right.$ and $\left.R_{\text {dif, elec }}\right)$ are the first parameters adjusted with the iterative fitting process explained hereafter. Only the part of the tests where all the studied
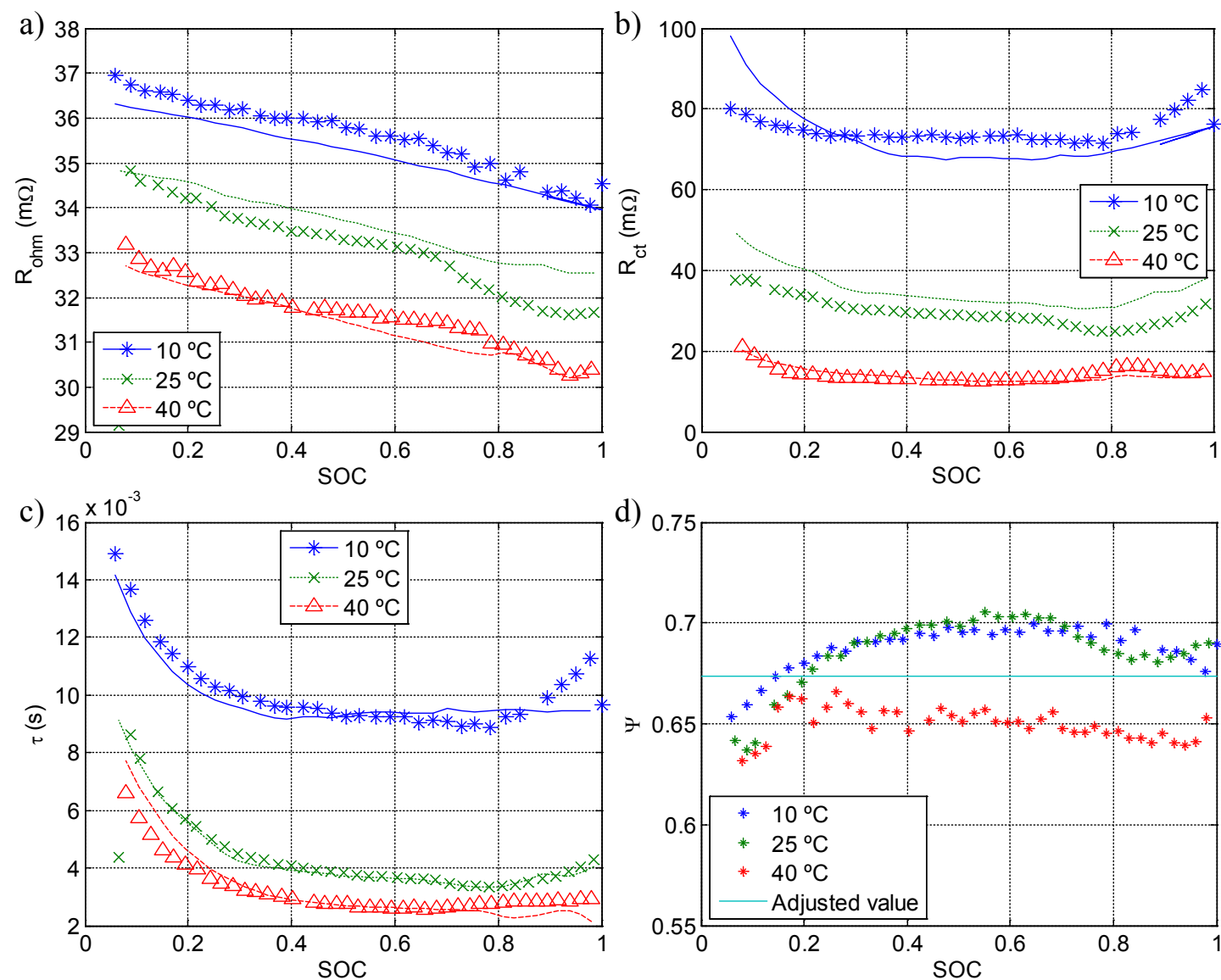

Fig. 8: Calculated parameters (markers) and fitting curves (solid lines): $R_{o h m}(\mathrm{a}), R_{c t}$ (b), $\tau$ (c) and $\Psi(\mathrm{d})$. 
phenomena are ${ }^{\circ}$ stabilised is used in order to achieve a good fitting of these resistances. Type $a$ experiments include discharge curves with different values of $i$ and $T$, which will be used to fit $R_{d i f, \text { mem. }}$ Since $v_{O C}, R_{o h m}$ and $R_{c t}$ are already known; the dotted curve from Fig. 9 can be calculated. As the first iteration step, the whole voltage drop measured in the central part of the discharge process will be assumed to be caused by $R_{d i f, m e m}$. As next step of the fitting process, the first approximate value of $R_{\text {dif, elec }}$ is calculated using the last part of the discharge process, where this resistance has its largest effect due to the shape of the discharge curve. This diffusion process in the electrodes is described by Eq. (40). At the end of the constantcurrent discharges from type $a$ experiments, diffusion phenomena are stabilized, the value of $S O C$ at these points is known and, therefore, $S O C_{\text {sur }}$ can be calculated using the equivalent circuit shown in Fig. 2 (b). The first approximate value of $R_{\text {dif, elec }}$ is obtained, and the process

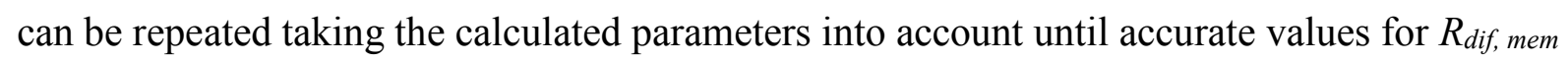
and $R_{\text {dif, elec }}$ are obtained. The values obtained for these variables after three iterations are shown in Fig. 10 (a) and (c). The expression of $R_{\text {dif, mem }}$ as a function of $T$, defined in Eq. (33), is fitted to these data as shown in Fig. 10 (a). Similarly, Eq. (42) is fitted to the values of $R_{\text {dif, elec }}$ as shown in Fig. 10 (c). The values obtained for the parameters are summarized in Table 1.

After the fitting of both diffusion resistances, the fitting of their associated capacitances is tackled. Capacitance $C_{d i f}$, mem can be studied with the stabilization process after each charge and discharge pulse in type $b$ experiments. The diffusion time constant is calculated for different SOC and $T$, the values for $C_{d i f, \text { mem }}$ shown in Fig. 10 (b) are obtained, and the independence of $C_{d i f, m e m}$ in relation to the SOC is confirmed. Neither has operating temperature $(T)$ a noticeable effect on the value of $C_{d i f, \text { mem }}$. Therefore, $C_{d i f, \text { mem }}$ is assumed to have the constant value shown in Table 1.

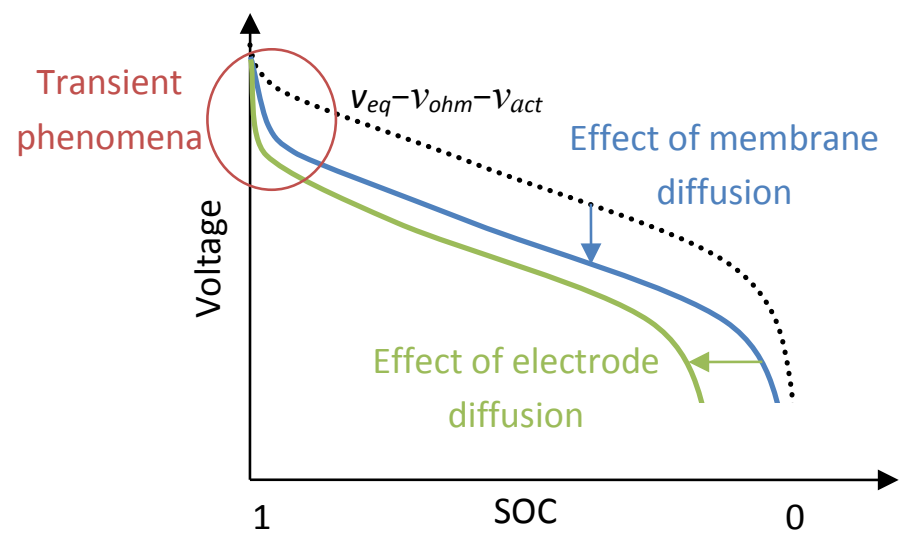

Fig. 9: Schematic representation of the voltage drops arising from each diffusion phenomenon during a constant-current battery discharge. 
The last parameter to be fitted is $C_{d i f, e l e c}$, which governs the slowest phenomenon taken into account in this model. The final parts of the type $a$ experiments (the constant voltage part of $\mathrm{CC}-\mathrm{CV}$ charges) are used to characterize its time constant. Considering that the electrode diffusion is in steady state when the charge mode is changed from $\mathrm{CC}$ to $\mathrm{CV}$, its time constant can be measured during the $\mathrm{CV}$ phase. The value of $C_{d i f \text {, elec }}$ for each test can be calculated, as shown in Fig. 10 (d). The fitting of Eq. (43) to these data provide the parameters shown in Table 1.
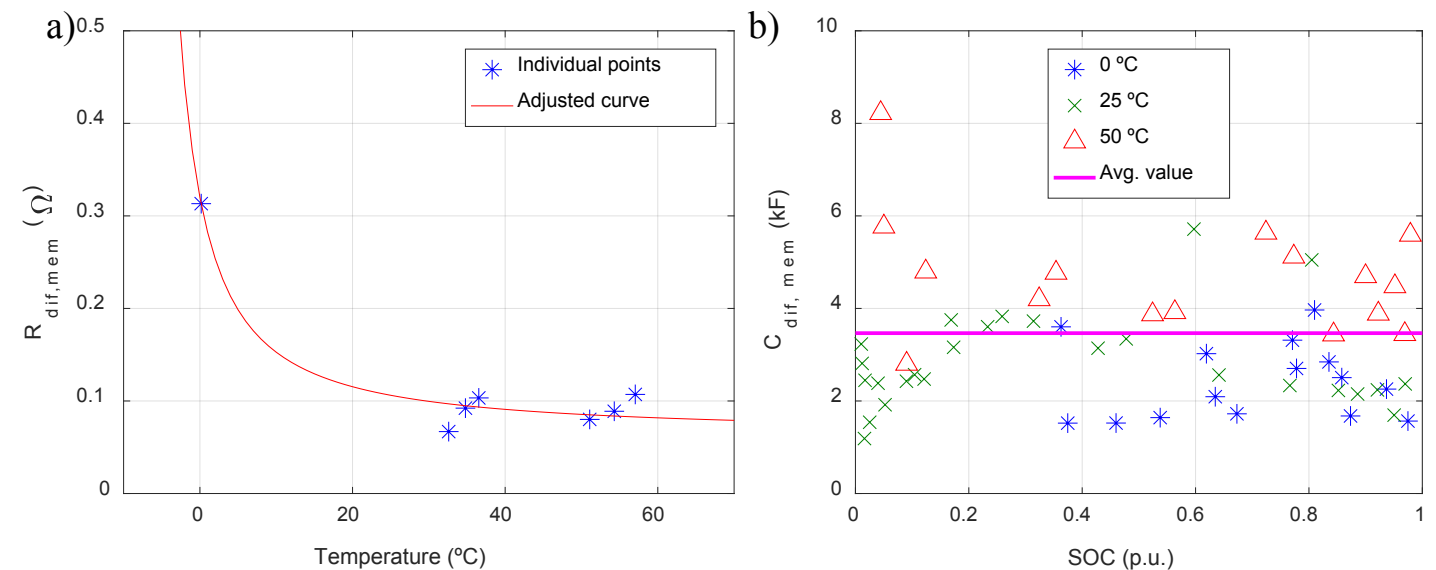

c)

d)
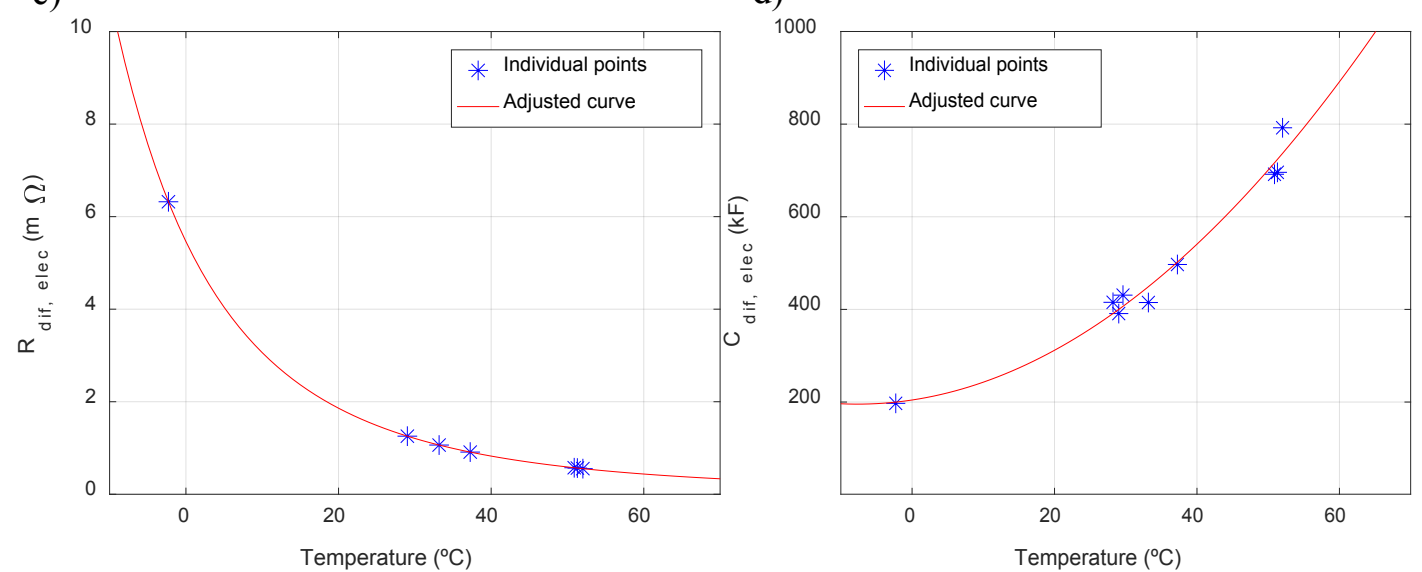

Fig. 10: Calculated parameters (markers) and fitting curves (solid lines) obtained for the diffusion processes: $R_{d i f, \text { mem }}(\mathrm{a}), C_{d i f, \text { mem }}(\mathrm{b}), R_{d i f, \text { elec }}(\mathrm{c})$ and $C_{d i f, \text { elec }}(\mathrm{d})$. 
Table 1. Model parameters.

\begin{tabular}{|c|c|c|c|c|c|c|c|c|c|}
\hline Phenomena & Eq. & Parameter & Value & Unit & Phenomena & Eq. & Parameter & Value & Unit \\
\hline Capacity & & C & 41 & $\mathrm{Ah}$ & \multirow{6}{*}{$\begin{array}{l}\text { Charge } \\
\text { transfer }\end{array}$} & 29 & $\Psi$ & 0.673 & \\
\hline \multirow{3}{*}{$\begin{array}{l}\text { Coulombic } \\
\text { efficiency }\end{array}$} & \multirow{3}{*}{19} & $\eta_{c, 0}$ & 0.9922 & & & \multirow{3}{*}{33} & $a_{0}$ & 0.0174 & $\mathrm{~s}$ \\
\hline & & $\eta_{c, T}$ & $2.08 \cdot 10^{-4}$ & ${ }^{\circ} \mathrm{C}^{-1}$ & & & $a_{1}$ & $-8.7 \cdot 10^{-4}$ & $\mathrm{~s}^{\circ} \mathrm{C}^{-1}$ \\
\hline & & $\eta_{c, i}$ & $-4.2 \cdot 10^{-5}$ & $A^{-1}$ & & & $a_{2}$ & $1.23 \cdot 10^{-5}$ & $\mathrm{~s}^{\circ} \mathrm{C}^{-2}$ \\
\hline \multirow{9}{*}{$\begin{array}{l}\text { Equilibrium } \\
\text { voltage }\end{array}$} & 20 & $U_{b a t}^{0}$ & $108.4 \mathrm{~V}$ & $\mathrm{~V}$ & & \multirow{2}{*}{42} & & & $\mathrm{~s}$ \\
\hline & \multirow{8}{*}{5} & $A_{0}$ & 54.96 & & & & $c$ & -7.18 & \\
\hline & & $A_{1}$ & -9.33 & & \multirow{4}{*}{$\begin{array}{l}\text { Membrane } \\
\text { diffusion }\end{array}$} & \multirow{4}{*}{34} & $K_{d i f, m e m}$ & 0.021 & $\mathrm{~A}^{-1}$ \\
\hline & & $A_{2}$ & 15.6 & & & & & 19.7 & ${ }^{\circ} \mathrm{C}$ \\
\hline & & $A_{3}$ & -4.732 & & & & $T_{0, \text { dif,mem }}$ & -13 & ${ }^{\circ} \mathrm{C}$ \\
\hline & & $A_{4}$ & -7.13 & & & & $C_{d i f, \text { mem }}$ & 3466 & F \\
\hline & & $A_{5}$ & -8.117 & & \multirow{6}{*}{$\begin{array}{l}\text { Electrode } \\
\text { diffusion }\end{array}$} & & $K_{\text {dif,elec }}$ & $1.37 \cdot 10^{-5}$ & $\mathrm{~A}^{-1}$ \\
\hline & & $A_{6}$ & 33.19 & & & 44 & $b_{\text {dif,elec }}$ & 468.2 & ${ }^{\circ} \mathrm{C}$ \\
\hline & & $A_{7}$ & -20.19 & & & & $T_{0, \text { dif,elec }}$ & -74.9 & ${ }^{\circ} \mathrm{C}$ \\
\hline \multirow{2}{*}{$\begin{array}{c}\text { Faradaic } \\
\text { current }\end{array}$} & \multirow{2}{*}{25} & $E_{A}$ & 41 & $\mathrm{~kJ} \cdot \mathrm{mol}^{-1}$ & & \multirow{3}{*}{45} & $a_{\text {elec }}$ & 119.3 & $\mathrm{~A} \cdot \mathrm{s} \cdot \mathrm{K}^{-}$ \\
\hline & & $A \cdot k_{0}^{0}$ & $1.95 \cdot 10^{-9}$ & $\mathrm{~m}^{2} \mathrm{~s}^{-1}$ & & & $b_{\text {elec }}$ & $-6 \cdot 10^{-4}$ & $\mathrm{~A} \cdot \mathrm{s} \cdot \mathrm{K}^{-1}$ \\
\hline \multirow{3}{*}{$\begin{array}{c}\text { Ohmic } \\
\text { phenomena }\end{array}$} & \multirow{3}{*}{23} & $R_{\text {ohm }, 0}$ & 73.58 & $\mathrm{~m} \Omega$ & & & $c_{\text {elec }}$ & $7.67 \cdot 10^{6}$ & $\mathrm{~A} \cdot \mathrm{s}$ \\
\hline & & $R_{o h m, T}$ & -0.1292 & $\mathrm{~m} \Omega \mathrm{K}^{-1}$ & & & & & \\
\hline & & $R_{\text {ohm }, S O C}$ & -2.849 & $\mathrm{~m} \Omega$ & & & & & \\
\hline
\end{tabular}

\section{6.- Comparison of experimental and simulated results}

\subsection{Full charge and discharge cycles}

The first set of experiments proposed to validate the good performance of the battery model consists in full charge-discharge cycles with different current and temperature values through the entire operating range $\left(10{ }^{\circ} \mathrm{C}, 35^{\circ} \mathrm{C}\right.$, and $\left.55^{\circ} \mathrm{C}\right)$. Discharge currents of $1 \mathrm{C}, 3 \mathrm{C}$, and $5 \mathrm{C}$ are analyzed, as shown in Fig. 11 (a), (b), and (c) respectively. The battery is charged following the standard $\mathrm{CC}-\mathrm{CV}$ procedure advised by the manufacturer, with a $\mathrm{CC}$ current of $1 \mathrm{C}$, as shown in Fig. 11 (d). The measured voltage is represented in this figures with markers, while the solid line represents the voltage predicted by the model. In the discharge tests (Fig. 11 (a), (b), and (c)) the battery voltage is revealed to decrease for decreasing temperatures, given that 
ohmic and charge transfer losses are increased. Especially remarkable is the voltage decrease measured with $T=10^{\circ} \mathrm{C}$. It limits the maximum battery discharge to around $65 \%$ of its nominal charge when $i=1 \mathrm{C}$ or $i=3 \mathrm{C}$ (Fig. 11 (a) and (b)). Likewise, the charge experiments shown in Fig. 11 (d) demonstrate a reduced CC charge provoked by a low temperature, which means an increased time for battery charge. It is also provoked by an increase in the ohmic and activation losses.

The model herein proposed accurately predicts the battery performance facing any of these constant-current requirements at any of the studied operating temperatures. A visual analysis of the graphs presented in Fig. 11 reveals that the model fails to predict the exact shape of the voltage curves for the most extreme situations $\left(T=10^{\circ} \mathrm{C}\right.$ or $\left.i=5 \mathrm{C}\right)$. However, each of the root mean square errors (RMSEs) obtained with the model simulations (see Table 2) is lower than $3 \mathrm{~V}$, which is considered a reasonable accuracy for the modeling of a battery with a rated voltage of $133 \mathrm{~V}$.
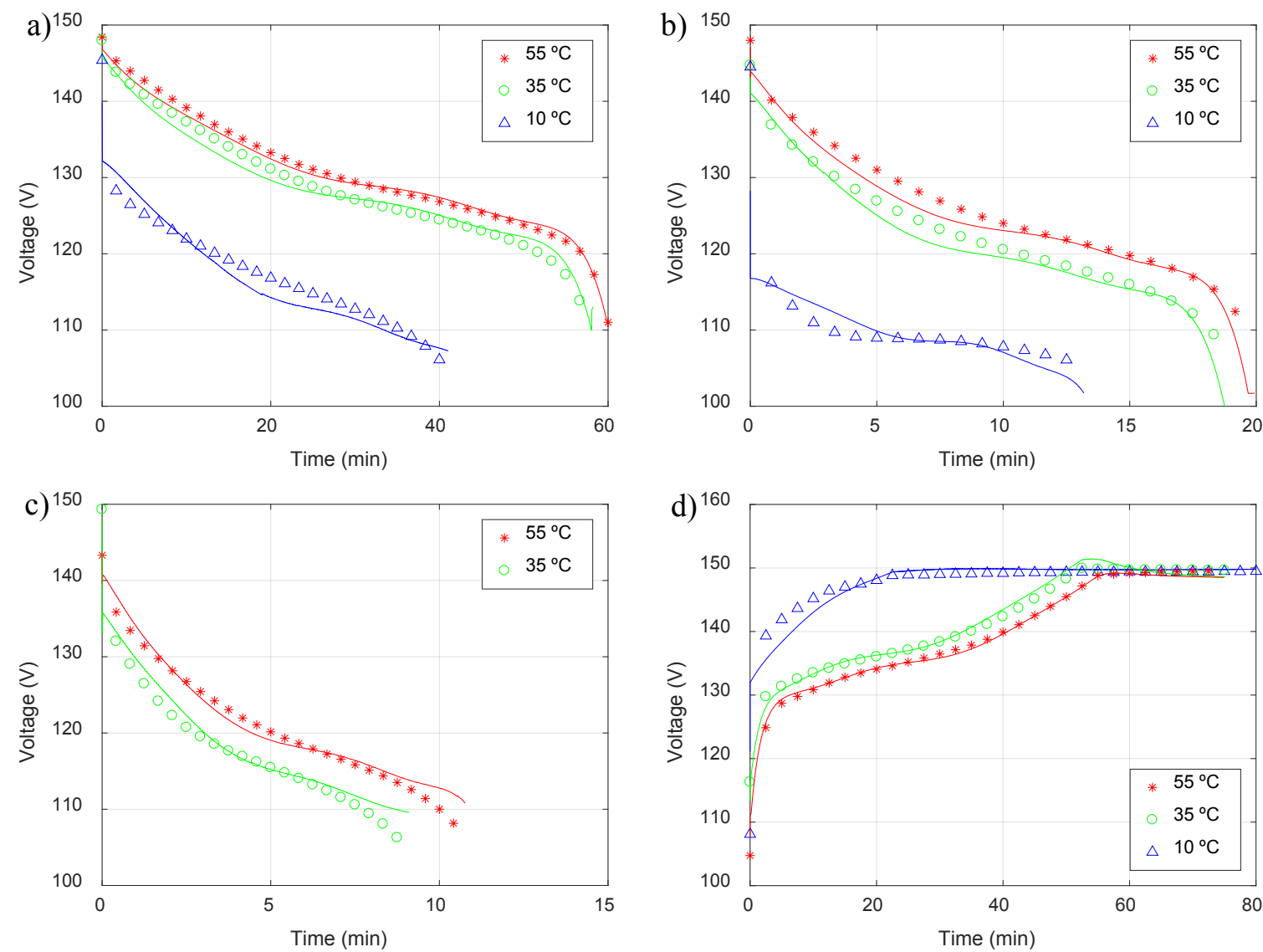

Fig. 11: Full cycle experiments: experimental measurements (markers) and model prediction (solid lines). Discharges with current $40 \mathrm{~A}$ (a), $120 \mathrm{~A}$ (b) and $200 \mathrm{~A}$ (c) and charges with $40 \mathrm{~A}(\mathrm{~d})$. 
Table 2. RMSE of the model during full charge and discharge experiments.

\begin{tabular}{lccc}
\cline { 2 - 4 } RMSE (V) & $10^{\circ} \mathrm{C}$ & $35^{\circ} \mathrm{C}$ & $55^{\circ} \mathrm{C}$ \\
\hline Discharge 40 A & 2.77 & 1.40 & 0.48 \\
Discharge 120 A & 2.63 & 2.94 & 2.69 \\
Discharge 200 A & -- & 2.46 & 2.18 \\
Charge 40 A & 1.47 & 1.12 & 0.22 \\
\hline
\end{tabular}

\subsection{Dynamic performance}

The battery was subjected to sinusoidal currents with an amplitude of $25 \mathrm{~A}$ and frequencies of $1 \mathrm{~Hz}$ and $100 \mathrm{~Hz}$. Furthermore, a sinusoidal current with an amplitude of $12.5 \mathrm{~A}$ and a frequency of $500 \mathrm{~Hz}$ is included. The ambient temperature is $23 \pm 1^{\circ} \mathrm{C}$, and the experiments are repeated with $\mathrm{SOC}=0.8$ and $\mathrm{SOC}=0.25$. The current - voltage $(i-v)$ relationships at each frequency are shown in Fig. 12 for $\mathrm{SOC}=0.8$ (a) and $\mathrm{SOC}=0.25$ (b). The effect of the double-layer is noted on these graphs, specifically for the $i-v$ relationship with a frequency of $100 \mathrm{~Hz}$, since this is the frequency where charge-transfer phenomena are noticeable. Furthermore, the slope of the $i-v$ curves decreases with increasing frequency due to the reduction of the equivalent battery resistance. A slightly higher slope of the curves with $S O C=0.25$ is also appreciated when compared to $S O C=0.8$. This effect is due to the
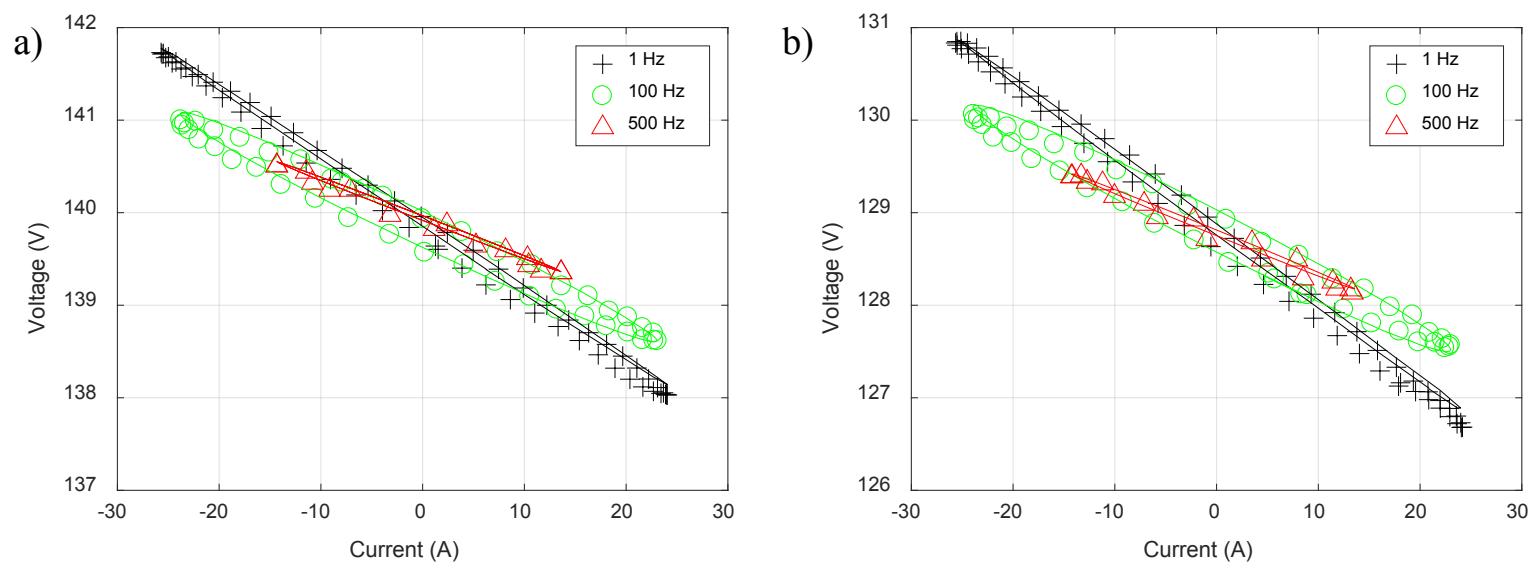

Fig. 12: $i-v$ relationships: experimental measurements (markers) and model prediction (solid lines) with $\mathrm{SOC}=0.8(\mathrm{a})$ and $\mathrm{SOC}=0.25(\mathrm{~b})$. 
increase in $R_{o h m}$ and $R_{c t}$ measured in Subsection 5.3. The validation for this dynamic operation is considered satisfactory, since RMSE values are lower than $90 \mathrm{mV}$ for $\mathrm{SOC}=0.8$ and lower than $100 \mathrm{mV}$ for $\mathrm{SOC}=0.25$.

\subsection{Operation of the battery in a real microgrid}

The battery model is validated herein while working in a real-life operating environment consisting of an electrical microgrid located at the UPNA [60]. The microgrid comprises a hybrid wind-photovoltaic renewable generating system and a storage system based on a lithium-ion battery. This microgrid is used to feed a five-member family home as shown in Fig. 13. The PV generator has a power of $4 \mathrm{kWp}$, while the wind power generator has $6 \mathrm{~kW}$. Likewise, the microgrid also allows for the energy exchange with the electricity grid. Due to the energy management strategies implemented in the microgrid, there is a daily coupling between the renewable power generation and the daily home consumption, while the annual fluctuations are absorbed by the electricity grid. In this way, the storage system guarantees a minimum power exchange with the electricity grid, keeping any inconvenience related to renewable energy generation away from the grid.

The proposed model is validated for the battery working as the storage system for this microgrid. For this purpose, an experimental validation with data from $11^{\text {th }}$ of April, 2014, between 15:00 $\mathrm{h}$ and 23:00 $\mathrm{h}$ is presented herein. There was no energy exchange with the grid during this time span. The power generated by the photovoltaic modules $\left(P_{p v}\right)$ and by the wind turbine $\left(P_{\text {wind }}\right)$ are shown in Fig. 14 (a). $P_{p v}$ has its maximum value at the beginning of the test and decreases to 0 at around 20:30 h. It was also a windy day, and the notable peaks of $P_{\text {wind }}$

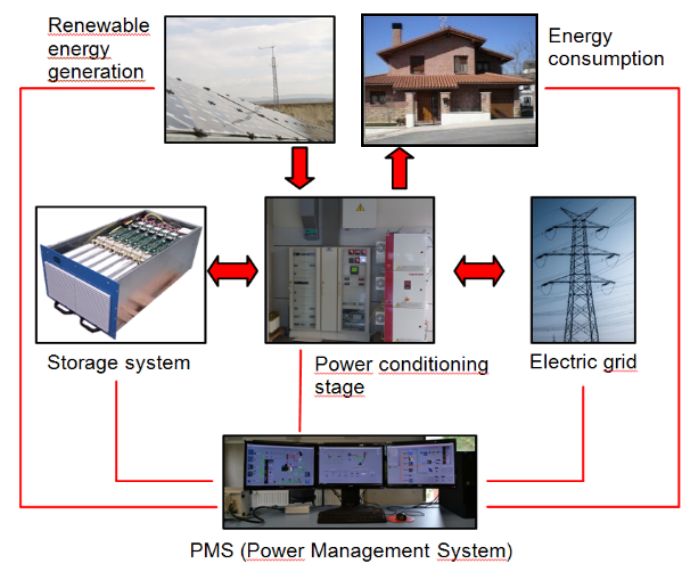

Fig. 13: Schematic diagram of the electric microgrid. 
are due to the usual gusty behavior of the wind. The power consumed in the family home $\left(P_{c o n}\right)$ is also shown in Fig. 14 (a). It has a mean value around $1.5 \mathrm{~kW}$ at the beginning of the test and reaches a peak of $2.6 \mathrm{~kW}$ at around 20:00 h, coinciding with the cooking of the evening meal.

The power profile that needs to be assumed by the battery is equivalent to the values of $v$ and $i$ shown in Fig. 14 (b). The top graph in Fig. 14 (b) shows the battery current (i). The bottom graph shows the battery voltage $(v)$, with a maximum value of $150 \mathrm{~V}$ and a minimum of $126 \mathrm{~V}$. The results obtained with the model show an adequate and reliable behaviour compared to the voltage measured during the experiment, with an RMSE in the voltage prediction of $0.627 \mathrm{~V}$. Therefore, it can be concluded that the model is accurate when simulating real-time operating environments.

\section{7.- Conclusion}

This paper reports on an equivalent-circuit model for lithium-ion batteries, the relationship of its parameters with the underlying physical phenomena that determine its performance, and the methodology to adjust the model parameters to a particular battery. Subsequently, the test procedure designed for the fitting process is explained and particularized for a graphite-NMC lithium-ion battery. The model parameters are adjusted to this commercial battery, and the accuracy of the model is finally validated in three scenarios.
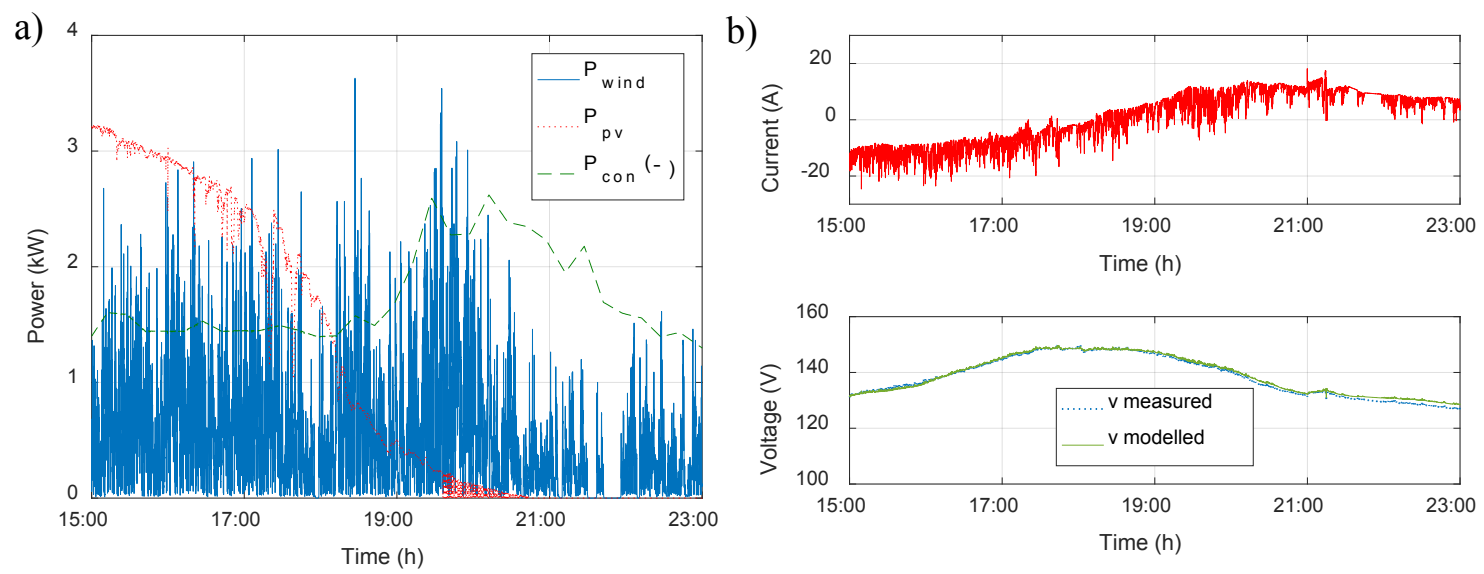

Fig. 14: Experiment and simulation of the Li-ion battery integration into the microgrid with data from $11^{\text {th }}$ of April, 2014. Power generated by the wind turbine $\left(P_{\text {wind }}\right)$, by the photovoltaic system $\left(P_{p v}\right)$ and consumed $\left(P_{c o n}\right)($ a) and battery current and voltage (b). 
The configuration of the battery model is principally based on the following phenomena: equilibrium potential of the electrochemical reaction, activation potential, double-layer effect, ohmic phenomena, diffusion through the electrodes and membrane, and SEI partial disintegration and formation. These phenomena are simplified and grouped to build the equivalent-circuit model which can predict the battery voltage taking the current and temperature as input variables. This link between the physical principles and the electrical performance can be exploited by battery designers willing to improve the electrical behavior of the battery, as well as by battery users who want to get the most from their battery while preserving its lifetime. No catastrophic failure events - like lithium plating, SEI breakdown or thermal runaway - are herein modeled. Therefore, the model is valid for the regular temperature, voltage and current ranges of the battery that entail no catastrophic failures

The parameter fitting strategy and the proposed tests to calculate the model parameters, which include constant-current charges and discharges, stepped charges and discharges, and electrochemical impedance spectroscopies, are detailed. These experiments are performed with a commercial $40 \mathrm{Ah}, 133 \mathrm{~V}$ battery, and the obtained results are summarized in a table. Besides, three validation scenarios are presented to prove the proper performance of the model. The first validation experiments consist of standard constant-current charges and discharges with different current requirements and temperature conditions. They prove the ability of the model to predict the battery behavior along the whole SOC range, subject to high power and with various operating temperatures. The second set of validation experiments are standard sinusoidal current tests, which show the accuracy of the model for currents ranging in a wide amplitude range both for high and low frequencies. Finally, the last test validates the battery model in a real operating environment. With this aim, the battery is integrated into an electrical microgrid and validated during 10 hours, obtaining an RMSE for the battery voltage of $0.627 \mathrm{~V}$.

Due to the model accuracy and stability shown during this study, this model can be considered a useful tool for the sizing, control, and performance analysis of an electrical system which includes a Li-ion battery.

\section{Acknowledgements}

We would like to acknowledge the support of the Spanish State Research Agency (AEI) and FEDER-UE under grants DPI2013-42853-R, DPI2016-80641-R and DPI2016-80642-R; of Government of Navarra through research project PI038 INTEGRA- 
RENOVABLES; and the FPU Program of the Spanish Ministry of Education, Culture and Sport (FPU13/00542).

\section{References}

[1] M. Stadler, G. Cardoso, S. Mashayekh, T. Forget, N. DeForest, A. Agarwal, A. Schönbein, Value streams in microgrids: A literature review. Applied Energy 162 (2016) 980-989.

[2] M. Soshinskaya, W. Crijns-Graus, J. van der Meer, J. Guerrero, Application of a microgrid with renewables for a water treatment plant. Applied Energy 134 (2014) 2034.

[3] R. Velik, P. Nicolay, Grid-price-dependent energy management in microgrids using a modified simulated annealing triple-optimizer. Applied Energy 130 (2014) 384-395

[4] E. Umeozor, M. Trifkovic, Operational scheduling of microgrids via parametric programming. Applied Energy 180 (2016) 672-681

[5] IRENA - International Renewable Energy Agency, Battery Storage for Renewables: Market Status and Technology Outlook, Abu Dhabi, 2015.

[6] IEC - International Electrochemical Commission, Electrical Energy Storage, Geneva, 2011.

[7] B. Diouf, R. Pode, Potential of lithium-ion batteries in renewable energy, Renew. Energy. 76 (2015) 375-380.

[8] X. Li, H. Huang, A. Faghri, Modeling study of a Li-O 2 battery with an active cathode, Energy 81 (2015) 489-500.

[9] S. Tippmann, D. Walper, L. Balboa, B. Spier, W.G. Bessler, Low-temperature charging of lithium-ion cells part I: Electrochemical modeling and experimental investigation of degradation behavior, J. Power Sources. 252 (2014) 305-316.

[10] A. Salvadori, D. Grazioli, M.G.D. Geers, Governing equations for a two-scale analysis of Li-ion battery cells, Int. J. Solids Struct. (2015) 1-20.

[11] L. Gu, D. Xiao, Y.-S. Hu, H. Li, Y. Ikuhara, Atomic-Scale Structure Evolution in a Quasi-Equilibrated Electrochemical Process of Electrode Materials for Rechargeable Batteries, Adv. Mater. (2015).

[12] T. F. Fuller, M. Doyle, J. Newman, Relaxation Phenomena in Lithium-Ion-Insertion Cells. J. Electrochem. Soc., 141, 4 (1994) 982-990.

[13] V. Srinivasan, J. Newman, Discharge Model for the Lithium Iron-Phosphate Electrode. J. Electrochem. Soc-, 151,10 (2004) A1517-A1529. 
[14] J. Newman, Optimization of Porosity and Thickness of a Battery Electrode by Means of a Reaction-Zone Model, J. Electrochem. Soc., 142, 1 (1995) 97-101.

[15] X. Li, S.-Y. Choe, W.T. Joe, A reduced order electrochemical and thermal model for a pouch type lithium ion polymer battery with $\mathrm{LiNi}_{\mathrm{x}} \mathrm{Mn}_{\mathrm{y}} \mathrm{Co}_{1-\mathrm{x}-\mathrm{y}} \mathrm{O}_{2} / \mathrm{LiFePO}_{4}$ blended cathode, J. Power Sources. 294 (2015) 545-555.

[16] S. Liu, J. Jiang, W. Shi, Z. Ma, L. Yi Wang, H. Guo, Butler - Volmer-Equation-Based Electrical Model for High-Power Lithium Titanate Batteries Used in Electric Vehicles, IEEE Trans. Ind. Electron. 62 (2015) 7557-7568.

[17] A. Berrueta, V. Irigaray, P. Sanchis, A. Ursúa, Lithium-ion battery model and experimental validation, in: EPE'15 ECCE Eur., pp. 1-8.

[18] W. Waag, C. Fleischer, D. U. Sauer, Critical review of the methods for monitoring of lithium-ion batteries in electric and hybrid vehicles. J. Power Sources. 258 (2014) 321339.

[19] Y. Li, C. Wang, J. Gong, A combination Kalman filter approach for State of Charge estimation of lithium-ion battery considering model uncertainty, Energy 109 (2016) 933-946

[20] Y. Wang, C. Liu, R. Pan, Z. Chen, Modeling and state-of-charge prediction of lithiumion battery and ultracapacitor hybrids with a co-estimator, Energy 121 (2017) 739-750.

[21] C. Zhang, W. Allafi, Q. Dinh, P. Ascencio, J. Marco, Online estimation of battery equivalent circuit model parameters and state of charge using decoupled least squares technique, Energy. 142 (2018) 678-688.

[22] Y. Wang, D. Yang, X. Zhang, Z. Chen, Probability based remaining capacity estimation using data-driven and neural network model, J. Power Sources. 315 (2016) 199-208.

[23] G. Dong, X. Zhang, C. Zhang, Z. Chen, A method for state of energy estimation of lithium-ion batteries based on neural network model, Energy 90 (2015) 879-888.

[24] X. Hu, S. Li, H. Peng, A comparative study of equivalent circuit models for Li-ion batteries, J. Power Sources. 198 (2012) 359-367.

[25] Q. K. Wang, Y. J. He, J. N. Shen, Z. F. Ma, G. B. Zhong, A unified modeling framework for lithium-ion batteries: An artificial neural network based thermal coupled equivalent circuit model approach, Energy, 138 (2017) 118-132.

[26] T. Feng, L. Yang, X. Zhao, H. Zhang, J. Qiang, Online identification of lithium-ion battery parameters based on an improved equivalent-circuit model and its implementation on battery state-of-power prediction, J. Power Sources. (2015).

[27] S. Nejad, D.T. Gladwin, D.A. Stone, A systematic review of lumped-parameter equivalent circuit models for real-time estimation of lithium-ion battery states, J. Power Sources. 316 (2016) 183-196. 
[28] C. Fleischer, W. Waag, H.-M. Heyn, D.U. Sauer, On-line adaptive battery impedance parameter and state estimation considering physical principles in reduced order equivalent circuit battery models part 2 . Parameter and state estimation, J. Power Sources. 262 (2014) 457-482.

[29] Z. Deng, Z. Deng, L. Yang, Y. Cai, X. Zhao, Implementation of reduced-order physicsbased model and multi-parameters identification strategy for lithium-ion battery, Energy. 138 (2017) 509-519.

[30] X. Zhang, Y. Wang, D. Yang, Z. Chen, An on-line estimation of battery pack parameters and state-of-charge using dual filters based on pack model, Energy 115 (2016) 219-229.

[31] M. Chen, G.A. Rincón-Mora, Accurate Electrical Battery Model Capable of Predicting Runtime and I - V Performance, IEEE Trans. ENERGY Convers. 21 (2006) 504-511.

[32] H. He, R. Xiong, X. Zhang, F. Sun, J. Fan, State-of-Charge Estimation of the LithiumIon Battery Using an Adaptive Extended Kalman Filter Based on an Improved Thevenin Model, IEEE Trans. Veh. Technol. 60 (2011) 1461-1469.

[33] B.Y. Liaw, G. Nagasubramanian, R.G. Gungst, D.H. Doughty, Modeling of lithium ion cells - A simple equivalent-circuit model approach, Solid State Ionics. 175 (2004) 835839.

[34] D.K. Karthikeyan, G. Sikha, R.E. White, Thermodynamic model development for lithium intercalation electrodes, J. Power Sources. 185 (2008) 1398-1407.

[35] R. Kanno, Y. Kawamoto, Carbon Fiber as a Negative Electrode in Lithium Secondary Cells, J. Electrochem. Soc. 139 (1992) 3397-3404.

[36] E. Tatsukawa, K. Tamura, Activity correction on electrochemical reaction and diffusion in lithium intercalation electrodes for discharge/charge simulation by single particle model, Electrochim. Acta. 115 (2014) 75-85.

[37] J. Sabatier, J.M. Francisco, F. Guillemard, L. Lavigne, M. Moze, M. Merveillaut, Lithium-ion batteries modeling: A simple fractional differentiation based model and its associated parameters estimation method, Signal Processing. 107 (2015) 290-301.

[38] J.-K. Park, The basic of battery chemistry, in: Princ. Appl. Lithium Second. Batter., Wiley-VCH: pp. 9-20.

[39] E. Barsoukov, J.R. Macdonald, Theory, in: J.W.\& Sons (Ed.), Impedance Spectroscopy. Theory, Experiment, and Application, 2nd ed.: pp. 27-128.

[40] K. Xu, Nonaqueous Liquid Electrolytes for Lithium-Based Rechargeable Batteries, Chem. Rev. 104 (2004) 4303-4417.

[41] International Standard. IEC 61427-2, (2015). 
[42] A. Farmann, W. Waag, A. Marongiu, D.U. Sauer, Critical review of on-board capacity estimation techniques for lithium-ion batteries in electric and hybrid electric vehicles, $\mathrm{J}$. Power Sources. 281 (2015) 114-130.

[43] J. Wang, P. Liu, J. Hicks-Garner, E. Sherman, S. Soukiazian,M. Verbrugge, H. Tataria, J. Musser, P. Finamore, Cycle-life model for graphite-LiFePO4 cells. J. Power Sources. 196 (2011) 3942-3948.

[44] Y. Zheng, M. Ouyang, L. Lu, J. Li, Z. Zhang, X. Li, Study on the correlation between state of charge and coulombic efficiency for commercial lithium ion batteries, J. Power Sources. 289 (2015) 81-90.

[45] D. Aurbach, B. Markovsky, I. Weissman, E. Levi, Y. Ein-Eli, On the correlation between surface chemistry and performance of graphite negative electrodes for Li ion batteries, Electrochim. Acta. 45 (1999) 67-86.

[46] J.R. Macdonald, M.K. Brachman, Linear-System Integral Transform Relations, Rev. Mod. Phys. 28 (1956) 393-422.

[47] J. Schrama, On the phenomenological theory of linear relaxation processes, University of Leiden, Netherlands, 1957.

[48] W. Dreyer, C. Guhlke, R. Müller, A new perspective on the electron transfer: recovering the Butler-Volmer equation in non-equilibrium thermodynamics. Physical Chemistry Chemical Physics, 18, 36 (2016) 24966-24983.

[49] J. Jiang, S. Liu, Z. Ma, L. Y. Wang, K. Wu, Butler-Volmer equation-based model and its implementation on state of power prediction of high-power lithium titanate batteries considering temperature effects, Energy 117 (2016) 58-72.

[50] A.J. Bard, L.F. Faulkner, Kinetiks of electrode reactions, in: J. W. \& Sons (Ed.), Electrochemical Methods. Fundamentals and Applications, 2nd ed.: pp. 87-136.

[51] S. Trasatti, Electrochemical Methods|Electrokinetics, in: Elsevier (Ed.), Encyclopedia of Electrochemical Power Sources: pp. 23-31.

[52] Y. Tsividis, J. Milios, A detailed look at electrical equivalents of uniform electrochemical diffusion using nonuniform resistance-capacitance ladders. Journal of Electroanalytical Chemistry, 707 (2013) 156-165.

[53] L. Zheng, L. Zhang, J. Zhu, G. Wang, J. Jiang, Co-estimation of state-of-charge, capacity and resistance for lithium-ion batteries based on a high-fidelity electrochemical model. Applied Energy 180 (2016) 424-434.

[54] G. Fan, K. Pan, M. Canova, J. Marcicki, X. Yang, Modeling of Li-Ion Cells for Fast Simulation of High C-Rate and Low Temperature Operations. J. Elect. Soc., 163, 5 (2016) A666-A676. 
[55] S. Ramachandran, A. Khandelwal, K. Hariharan, B. Kim, K. Kim. Rapid Analysis of Charging Profiles of Lithium Ion Batteries Using a Hybrid Simplified Electrochemical Model. J. Elect. Soc., 163, 6 (2016) A1101-A1111.

[56] N. Yang, X. Zhang, G. Li, State-of-charge estimation for lithium ion batteries via the simulation of lithium distribution in the electrode particles, J. Power Sources. 272 (2014) 68-78.

[57] E. Barsoukov, J.R. Macdonald, Fundamentals of Impedance Spectroscopy, in: J.W.\& Sons (Ed.), Impedance Spectroscopy Theory, Experiment, and Application., 2nd ed.: pp. 1-26.

[58] M.C. Smart, J.F. Whitacre, B. V. Ratnakumar, K. Amine, Electrochemical performance and kinetics of $\mathrm{Li}_{1+\mathrm{x}}\left(\mathrm{Co}_{1 / 3} \mathrm{Ni}_{1 / 3} \mathrm{Mn}_{1 / 3}\right)_{1-\mathrm{x}} \mathrm{O}_{2}$ cathodes and graphite anodes in lowtemperature electrolytes, J. Power Sources. 168 (2007) 501-508.

[59] B.Y. Liaw, E.P. Roth, R.G. Jungst, G. Nagasubramanian, H.L. Case, D.H. Doughty, Correlation of Arrhenius behaviors in power and capacity fades with cell impedance and heat generation in cylindrical lithium-ion cells, J. Power Sources. 119-121 (2003) $874-886$.

[60] J. Pascual, J. Barricarte, P. Sanchis, L. Marroyo, Energy management strategy for a renewable-based residential microgrid with generation and demand forecasting, Applied Energy. 158 (2015) 12-25. 